\title{
Recoilles Spin-Flip in the Magnetic Scattering of Cold Neutrons
}

\author{
P. H. HANDEL
}

Institut Max von Laue-Paul Langevin, 8046 Garching bei München

(Z. Naturforschg. 24 a, 1646-1659 [1969]; received 5 July 1969)

\begin{abstract}
The probability of magnetic and nuclear magnetic recoilless spin-flip scattering in magnetic crystals is calculated as a function of temperature. This is an elastic spin-flip scattering process in which the angular momentum is absorbed by the whole crystal. The probability of elastic scattering in a ferromagnet is proportional to the squared magnetization and plays the same part as the Debye-Waller factor in the Mössbauer effect.
\end{abstract}

Neutrons of low energy interacting with a magnetic crystal are subject to nuclear scattering as well as to magnetic scattering by the magnetic moment of the electronic shells. Each of the cross sections for these two major types of scattering is of the order of one barn. Nuclear scattering includes both scattering by nuclear forces (true nuclear scattering) and magnetic nuclear scattering which is due to the interaction of the magnetic moment of the neutron with that of the nucleus if the latter is present. Magnetic nuclear scattering amplitudes are about three orders of magnitude smaller than electronic ones. They include the nuclear magneton $\mu_{\mathrm{n}}$ squared, whereas ordinary magnetic scattering amplitudes depend linearly on $\mu_{\mathrm{n}}$, along with the Bohr-magneton $\mu_{\mathrm{B}}$. Thus nuclear magnetic scattering is usually neglected. As we shall see, at very low energies $\left(10^{-6}-10^{-7} \mathrm{eV}\right)$ nuclear spin-flip scattering is energetically forbidden under certain conditions. It is only in this case that nuclear magnetic scattering has to be taken into account.

Our major interest will be in elastic spin-flip processes in which the spin of the neutron is reversed and the crystal as a whole absorbs the angular momentum. This is analogous to elastic Bragg scattering or to recoilles $\gamma$-emission (Mössbauer-effect). However, in this case the scattering is recoilless with respect to the spin, which means that no spin-recoil should appear. By spin-recoil we mean the absorption of angular momentum by interal modes of the crystal: flipping of the spin of a nucleus, emission of a spin wave, etc.

As in the case of recoilless X-Ray scattering or the Mössbauer-effect we shall derive a temperature dependent probability for transitions occurring with-

Reprint requests to Dr. Peter H. HANDel, University of Missouri-St. Louis, Dept. of Physics, 8001 Natural Bridge, St. Louis, Mo 63 121, USA. out spin-recoil. It turns out that this probability is a monotonically decreasing function of the temperature, like the Debye-Waller factor.

We shall first derive the contribution of the nuclear magnetic scattering to the recoilless spin-flip amplitude.

\section{Scattering by Oriented Atomic Nuclei}

\subsection{The Spin-Orbit Transitions}

In an internal magnetic field $H$ the fractional number of nuclei with magnetic quantum number $m, g(m, T)$, is determined by a Boltzmann factor:

$$
g\left(m_{0}, T\right)=\frac{\exp \left\{-W\left(m_{0}\right) / k_{\mathrm{B}} T\right\}}{\sum_{m=-I}^{I} \exp \left\{-W(m) / k_{\mathrm{B}} T\right\} .}
$$

Here $W(m)=-\gamma_{n} \mu_{\mathrm{n}} H m=-\boldsymbol{b} \mathbf{I}$ is the orientation energy of the nuclear spin $I, \mu_{\mathrm{n}}$ the nuclear magneton, $\gamma_{\mathrm{n}}$ the gyromagnetic coefficient of the nucleus, $\gamma_{\mathrm{n}} \mu_{\mathrm{n}}$ the corresponding gyromagnetic factor, $\boldsymbol{b}=\gamma_{\mathrm{n}} \mu_{\mathrm{n}} \boldsymbol{H}, T$ the temperature and $k_{\mathrm{B}}$ Boltzmann's constant. Due to the large values of the field $H$ at the positions of the nuclei, in some crystals ${ }^{1}$ the nuclear orientation will be effective at temperatures below $100{ }^{\circ} \mathrm{K}$. The corresponding internal fields are $10^{6}$ Oe and more $\left(4 \pm 110^{6} \mathrm{Oe}\right.$ in $\mathrm{Th}^{159}$ at $77^{\circ} \mathrm{K}$ for instance).

The Hamiltonian of the neutron and the scattering nucleus is

$$
\begin{aligned}
& \mathcal{H}_{\mathrm{Nn}}=-\left(\hbar^{2} / 2 m_{\mathrm{N}}\right) \nabla^{2}-\boldsymbol{b} \mathbf{I}+(c+d \boldsymbol{I} \boldsymbol{s}) \delta \boldsymbol{r} \\
&+\left(\varepsilon / r^{3}\right)[3(\boldsymbol{I} \boldsymbol{r} / r)(\boldsymbol{s} \mathbf{r} / \boldsymbol{r})-\boldsymbol{I} \boldsymbol{s}] .
\end{aligned}
$$

We denote the spin of the neutron (all angular momenta are expressed in units of $\hbar$ ) by $\boldsymbol{s}$, the position vector of the neutron with respect to the nucleus 
by $\boldsymbol{r}$, and

$$
\begin{gathered}
c \equiv-\frac{2 \pi \hbar^{2}}{m_{\mathrm{N}}} \frac{a_{0} I+a_{1}(I+1)}{2 I+1}, \quad d \equiv \frac{8 \pi \varepsilon}{3}-(3) \\
-\frac{4 \pi \hbar^{2}}{m_{\mathrm{N}}} \frac{a_{1}-a_{0}}{2 I+1} \equiv \frac{8 \pi \varepsilon}{3}+d_{0}, \quad \varepsilon \equiv-\gamma_{\mathrm{N}} \gamma_{\mathrm{n}} \mu_{\mathrm{n}}^{2} .
\end{gathered}
$$

Here $a_{0}$ and $a_{1}$ are the nuclear scattering amplitudes corresponding to states with total spin $I-\frac{1}{2}$ and $I+\frac{1}{2}, \gamma_{\mathrm{N}}=1.91$ the gyromagnetic coefficient of the neutron, $m_{\mathrm{N}}$ the mass of the neutron and $\delta(\boldsymbol{r})$ the three dimensional Dirac delta function. The third term of Eq. (2) is essentially the nuclear interaction which is described with a Fermi-potential. The fourth term, together with that part of the preceding one which is proportional to $\varepsilon$, is the magnetic dipole-dipole interaction of the neutron and the nucleus.

The total spin projection $I_{z}+s_{z}$ is not a conserved quantity

$$
\begin{aligned}
& {\left[I_{z}+s_{z}, \mathcal{H}_{\mathrm{Nn}}\right]=-b_{x} I_{y}+b_{y} I_{x}} \\
& +\left(3 \varepsilon / r^{4}\right)\left[\left(I_{y} x-I_{x} y\right)(\boldsymbol{s} \boldsymbol{r} / \boldsymbol{r})\right. \\
& \left.+(\boldsymbol{I} \boldsymbol{r} / r)\left(s_{y} x-s_{x} y\right)\right] \neq 0
\end{aligned}
$$

nor is the orbital moment of the system $L_{z}$

$$
\begin{aligned}
{\left[L_{z}, \mathcal{H}_{\mathrm{Nn}}\right]=-\left(3 \varepsilon / r^{4}\right) } & {\left[\left(I_{y} x-I_{x} y\right)(\boldsymbol{s} \boldsymbol{r} / r)\right.} \\
+ & \left.(\mathbf{I r} / \boldsymbol{r})\left(s_{y} x-s_{x} y\right)\right] .
\end{aligned}
$$

If we choose the $z$-axis in the direction of $\boldsymbol{b}$, which is the direction of the internal field $\boldsymbol{H}$, the terms involving $b_{z}$ and $b_{y}$ will disappear from Eq. (4). Then $J_{z}=I_{z}+s_{z}+L_{z}$ is a conserved quantity. Note that in the absence of the last term of Eq. (2) both the total spin projection $I_{z}+s_{z}$ and $L_{z}$ are conserved.

The magnetic dipole-dipole interaction allows for transitions in which the total spin projection $I_{z}+s_{z}$ changes by 1 or 2 units. Let us call them spin-orbit (s.-o.) transitions. In a spin-orbit scattering process the orbital momentum projection of the initial state differs from that of the final state by the same amount as the change in $I_{z}+s_{z}$; the total angular momentum is thus conserved. The nuclear scattering described by the third term of Eq. (2) does not involve s.-o. processes.

There are certain s.-o. processes in which $I_{z}$ is constant. They simply yield a spin-flip scattering without spin-recoil. If in addition no phonons are produced, the spin-flip scattering is completely elas- tic. The recoilless spin-flip scattering (r.s.f.) by oriented nuclei is due only to the s.-o. processes generated by the magnetic dipole-dipole interaction of the neutron with the nucleus. The corresponding scattering amplitude will be very small, because $\varepsilon$ is a small parameter. We shall see in Sect. 2 that much larger r.s.f. amplitudes can arise from mag. netic scattering by the electronic shells. This is absent, however, if the electronic shells are magnetically compensated. We shall thus briefly examine the nuclear and nuclear-magnetic scattering amplitudes before turning to the main magnetic scattering.

\subsection{Nuclear Scattering Amplitudes}

In order to obtain the scattering amplitude in the first Born-approximation, we write Eq. (2) :

with

$$
\mathcal{H}_{\mathrm{Nn}}=\mathcal{H}_{\mathrm{Nn}}^{0}+V
$$

$$
\mathcal{H}_{\mathrm{Nn}}^{0}=-\left(\hbar^{2} / 2 m_{\mathrm{N}}\right) \nabla^{2}-\boldsymbol{b} \mathbf{I} \text {. }
$$

We obtain the first-order correction to the wavefunction, $\psi_{1}$, from the Schrödinger-equation

Here

$$
\left(\mathcal{H}_{\mathrm{Nn}}^{0}-E\right) \psi_{1}^{ \pm}=-V \psi_{0}^{ \pm} \text {. }
$$

$$
\begin{gathered}
\psi_{0}^{+}(\boldsymbol{r}, M, S)=\exp \left(i \boldsymbol{k}_{\mathrm{e}} \boldsymbol{r}\right) \Omega_{m_{\mathrm{a}}}(M) \omega_{1 / 2}(S), \\
\psi_{0}^{-}(\boldsymbol{r}, M, S)=\exp \left(i \boldsymbol{k}_{\mathrm{e}} \boldsymbol{r}\right) \Omega_{m_{\mathrm{0}}}(M) \omega_{-1 / 2}(S),
\end{gathered}
$$

describes the state in the absence of the interaction $V, \Omega_{m_{0}}(M)$ is the spinfunction of the nucleus with $I_{z}=m_{0}$ and $\omega_{ \pm 1 / 2}(S)$ that of the neutron with $s_{z}= \pm \frac{1}{2}$.

The calculation has been performed to second order ${ }^{1}$ but all s.-o. processes were omitted from the beginning. We shall use a similar approach here, limiting ourselves to first order but including all s.-o. processes. It turns out that for very low energies the second order correction is negligible, proportional to the wavevector of the neutrons.

The Green-function of Eq. (8) can be calculated from

$\left(\mathcal{H}_{\mathrm{Nn}}^{0}-E\right) G\left(\boldsymbol{r}, \boldsymbol{r}^{\prime}, M, M^{\prime}, S, S^{\prime}\right)=\delta\left(\boldsymbol{r}-\boldsymbol{r}^{\prime}\right) \delta_{M M^{\prime}} \delta_{S S^{\prime}}$.

With

$$
k(m)=\sqrt{k_{\mathrm{e}}{ }^{2}+\left(m-m_{0}\right) \cdot 2 m_{\mathrm{N}} b / \hbar^{2}}
$$

we obtain

$$
G\left(\boldsymbol{r}, \boldsymbol{r}^{\prime}, M, M^{\prime}, S, S^{\prime}\right)=\frac{m_{\mathrm{N}}}{2 \pi \hbar^{2}} \sum_{m} \sum_{s} \frac{\exp \left\{i k(m)\left|\boldsymbol{r}-\boldsymbol{r}^{\prime}\right|\right\}^{\prime}}{\left|\boldsymbol{r}-\boldsymbol{r}^{\prime}\right|} \Omega_{m}(M) \Omega_{\mathrm{m}}^{*}\left(M^{\prime}\right) \omega_{s}(S) \omega_{s}\left(S^{\prime}\right)
$$


This yields

$\psi_{1}^{ \pm}=\sum_{\mathbb{M}^{\prime}} \sum_{\mathrm{S}^{\prime}} \int \mathrm{d}^{3} \boldsymbol{r}^{\prime} G\left(\boldsymbol{r}, \boldsymbol{r}^{\prime}, M, M^{\prime}, S, S^{\prime}\right)$

$$
\cdot\left\{(c+\boldsymbol{I} \boldsymbol{s} d) \delta\left(\boldsymbol{r}^{\prime}\right)+\frac{\varepsilon}{r^{\prime 3}}\left[3\left(\boldsymbol{I} \frac{\boldsymbol{r}^{\prime}}{r^{\prime}}\right)\left(\boldsymbol{s} \frac{\boldsymbol{r}^{\prime}}{r^{\prime}}\right)-\boldsymbol{I} \boldsymbol{s}\right]\right\} \exp \left\{i \boldsymbol{k}_{\mathrm{e}} \boldsymbol{r}^{\prime}\right\} \Omega_{m_{\mathbf{l}}}\left(M^{\prime}\right) \omega_{ \pm 1 / 2}\left(S^{\prime}\right)
$$

Performing the integration we finally obtain

$$
\begin{aligned}
& \psi_{1}^{ \pm}(\boldsymbol{r}, M, S)=\left[f_{\mathrm{nf}}^{0 \pm} \omega_{ \pm 1 / 2}(S)+f_{\mathrm{f}}^{0 \pm} \omega_{ \pm} \omega_{ \pm 1 / 2}(S)\right]\left(e^{i k_{\mathrm{o}} r} / r\right) \Omega_{m_{\mathrm{o}}}(M) \\
& \quad+\left[f_{\mathrm{nf}}^{\prime \pm} \omega_{ \pm 1 / 2}(S)+f^{ \pm \pm} \omega_{ \pm 1 / 2}(S)\right]\left(e^{i k^{\prime} r} / r\right) \Omega_{m_{0}+1}(M)+\left[f_{\mathrm{nf}}^{\prime \prime} \omega_{ \pm 1 / 2}(S)+f_{\mathrm{f}}^{\prime \prime} \omega_{ \pm 1 / 2}(S)\right]\left(e^{i k^{\prime \prime} / r} / r\right) \Omega_{m_{\mathrm{o}}-1}(M),
\end{aligned}
$$

where the upper + and - signs correspond to the "up" and "down" spin states of the incident neutrons and

$$
\begin{aligned}
& f_{\mathrm{nf}}^{0 \pm}=\left(m_{\mathrm{N}} / 2 \pi \hbar^{2}\right)\left[c \pm \frac{1}{2} m_{0} d_{0} \pm 2 \pi \varepsilon m_{0} \sin ^{2} \theta_{0}\right] ; \\
& f_{\mathrm{f}}^{0 \pm}=\left(m_{\mathrm{N}} / 2 \hbar^{2}\right) \varepsilon m_{0} \sin 2 \theta_{0} ; \cos \theta_{0}=\boldsymbol{e}_{z} \cdot\left(\boldsymbol{k}_{0}-\boldsymbol{k}_{\mathrm{e}}\right) /\left|\boldsymbol{k}_{0}-\boldsymbol{k}_{\mathrm{e}}\right|,\left|\boldsymbol{k}_{0}\right|=\left|\boldsymbol{k}_{\mathrm{e}}\right|=k\left(m_{0}\right) ; \\
& f_{\mathrm{nf}}^{\prime \pm}= \pm\left(m_{\mathrm{N}} / 2 \hbar^{2}\right) \frac{1}{2} \varepsilon V\left(I-m_{0}\right)\left(I+m_{0}+1\right) \sin 2 \theta^{\prime} ; \cos \theta^{\prime}=\boldsymbol{e}_{z} \cdot\left(\boldsymbol{k}^{\prime}-\boldsymbol{k}_{\mathrm{e}}\right) /\left|\boldsymbol{k}^{\prime}-\boldsymbol{k}_{\mathrm{e}}\right|, k^{\prime}=k\left(m_{0}+1\right) ; \\
& f_{\mathrm{f}}^{\prime \pm}=\left(m_{\mathrm{N}} / 2 \pi \hbar^{2}\right) \sqrt{\left(I-m_{0}\right)\left(I+m_{0}+1\right)}\left[\frac{1}{2} d_{0}+\pi \varepsilon\left(1+\cos ^{2} \theta^{\prime}\right)\right] \\
& f_{\mathrm{f}}^{\prime}=-\left(m_{\mathrm{N}} / 2 \hbar^{2}\right) \varepsilon \sqrt{\left(I-m_{0}\right)} \overline{\left(I+m_{0}+1\right)} \sin ^{2} \theta^{\prime} ; \\
& f_{\mathrm{nf}}^{\prime \prime}= \pm\left(m_{\mathrm{N}} / 2 \hbar^{2}\right) \frac{1}{2} \varepsilon \sqrt{\left(I+m_{0}\right)\left(I-m_{0}+1\right)} \sin 2 \theta^{\prime \prime} ; \cos \theta^{\prime \prime}=\boldsymbol{e}_{z} \cdot\left(\boldsymbol{k}^{\prime \prime}-\boldsymbol{k}_{\mathrm{e}}\right) /\left|\boldsymbol{k}^{\prime \prime}-\boldsymbol{k}_{\mathrm{e}}\right|, k^{\prime \prime}=k\left(m_{0}-1\right) ; \\
& f_{\mathrm{f}}^{\prime \prime}+=-\left(m_{\mathrm{N}} / 2 \hbar^{2}\right) \varepsilon \sqrt{\left(I+m_{0}\right)\left(I-m_{0}+1\right)} \sin ^{2} \theta^{\prime \prime} ; \\
& f_{\mathrm{f}}^{\prime \prime}-=\left(m_{\mathrm{N}} / 2 \pi \hbar^{2}\right) \sqrt{\left(I+m_{0}\right)\left(I-m_{0}+1\right)}\left[\frac{1}{2} d_{0}+\pi \varepsilon\left(1+\cos ^{2} \theta^{\prime \prime}\right)\right] .
\end{aligned}
$$

The subscripts "nf" and "f" distinguish non-spin-flip and spin-flip amplitudes. The initial magnetic quantum number of the nucleus is $m_{0} . \boldsymbol{k}_{\mathbf{0}}, \boldsymbol{k}^{\prime}$ and $\boldsymbol{k}^{\prime \prime}$ are the wave-vectors of the outgoing waves, corresponding to the final nuclear states $m_{0}, m_{0}+1$ and $m_{0}-1$. Note that $f_{\mathrm{nf}}^{0+}, f_{\mathrm{f}}^{\prime+}$ and $f_{\mathrm{f}}^{\prime \prime}-$ are the dominant amplitudes which are allowed by the nonmagnetic nuclear interaction and which correspond to $\Delta L_{z}=0$. All other amplitudes are about 3 orders of magnitude smaller, involving s.-o. processes $\left(\Delta L=2, \Delta L_{z}= \pm 1, \pm 2\right)$ which are due only to the nuclear magnetic dipole-dipole interaction.

\subsection{Nuclear Cross-Sections}

If no other interaction is present, as in the absence of a magnetic moment of the electronic shells, we obtain from Eqs. (16) - (21) the following cross sections for the 6 channels involved (for any initial direction of polarization)

$$
\begin{aligned}
& \sigma_{\mathrm{nf}}^{0 \pm}=\left(m_{\mathrm{N}} / 2 \pi \hbar^{2}\right)^{2}\left[c^{2}+\frac{1}{4} m^{2} d_{0}^{2}+4 \pi^{2} \varepsilon^{2} m_{0}^{2} \sin ^{4} \theta_{0} \pm c m d_{0} \pm\left(2 c \pm \mathrm{m}_{0} d_{0}\right) 2 \pi \varepsilon m_{0} \sin ^{2} \theta_{0}\right] \\
& \sigma_{\mathrm{f}}^{0 \pm}(m)=\left(m_{\mathrm{N}} / 2 \hbar^{2}\right)^{2} \varepsilon^{2} m^{2} 2 \theta_{0} \\
& \sigma_{\mathrm{nf}}^{\prime \pm}(m)=\left(m_{\mathrm{N}} / 2 \hbar^{2}\right)^{2} \frac{1}{4} \varepsilon^{2}(I-m)(I+m+1) \sin ^{2} 2 \theta_{0} \operatorname{Re} \sqrt{1+2 m_{\mathrm{N}} b / \hbar^{2} k_{0}{ }^{2}} \\
& \sigma_{\mathrm{f}}^{\prime \pm}(m)=\left(m_{\mathrm{N}} / 2 \pi \hbar^{2}\right)^{2}(I-m)(I+m+1)\left[\frac{1}{4} d_{0}^{2}+\pi^{2} \varepsilon^{2}\left(1+\cos ^{2} \theta^{\prime \prime}\right)^{2}\right. \\
& \left.\quad+\pi d_{0} \varepsilon\left(1+\cos ^{2} \theta^{\prime}\right)\right] \operatorname{Re} \sqrt{1+2 m_{\mathrm{N}} b / \hbar^{2} k_{0}^{2}} \\
& \sigma_{\mathrm{f}}^{\prime}-(m)=\left(m_{\mathrm{N}} / 2 \hbar^{2}\right)^{2} \varepsilon^{2}(I-m)(I+m+1) \sin ^{4} \theta^{\prime} \operatorname{Re} \sqrt{1+2 m_{\mathrm{N}} b / \hbar^{2} k_{0}^{2}} \\
& \sigma_{\mathrm{nf}}^{\prime \prime \pm}(m)=\left(m_{\mathrm{N}} / 2 \hbar^{2}\right)^{2} \frac{1}{4} \varepsilon^{2}(I+m)(I-m+1) \sin ^{2} 2 \theta^{\prime \prime} \operatorname{Re} \sqrt{1-2 m_{\mathrm{N}} b / \hbar^{2} k_{0}}{ }^{2}
\end{aligned}
$$

1 K. Binder and H. RaUch, Nukleonik 11, 113 [1968]. 
$\sigma_{\mathrm{f}}^{\prime \prime}+(m)=\left(m_{\mathrm{N}} / 2 \hbar^{2}\right)^{2} \varepsilon^{2}(I+m)(I-m+1) \sin ^{4} \theta^{\prime \prime} \operatorname{Re} \sqrt{1-2 m_{\mathrm{N}} b / \hbar^{2} k_{0}{ }^{2}} ;$

$\sigma_{\mathrm{f}}^{\prime \prime}-(m)=\left(m_{\mathrm{N}} / 2 \pi \hbar^{2}\right)^{2}(I+m)(I-m+1)\left[\frac{1}{4} d_{0}^{2}+\pi^{2} \varepsilon^{2}\left(1+\cos ^{2} \theta^{\prime \prime}\right)^{2}\right.$

$$
\left.+\pi d_{0} \varepsilon\left(1+\cos ^{2} \theta^{\prime \prime}\right)\right] \operatorname{Re} \sqrt{1-2 m_{\mathrm{N}} b / \hbar^{2} k_{0}{ }^{2}} .
$$

Here we dropped the lower 0-index of $m$. Taking the real part of the square root which occurs in the $\sigma^{\prime}$ and $\sigma^{\prime \prime}$ cross sections involves a low energy cutoff in half of them. This happens if the reorientation of the nucleus is endoenergetic and the energy of the incoming neutrons is smaller than this reorientation energy. Then the transition is energetically forbidden, and only the s.-o. spin-flip transitions (23) are allowed at lower energies. The cutoff will be quite sharp. For most nuclei $\gamma_{N}<0$ and thus $b<0$ leading to cutoffs of the $\sigma^{\prime}$ cross sections only. Conversely, for nuclei with $\gamma_{\mathrm{N}}>0$, the $\sigma^{\prime \prime}$ 's will contain the low-energy cut-off. Typical values of the cut-off energy $b$ are very small; $b \approx 2 \cdot 10^{-5} \mathrm{eV}$ for $H=$ $4 \cdot 10^{7}$ Oe.

We denote by $p_{0 z}$ the component of the polarization of the incident neutrons in the $z$-direction. This is twice the mean $s_{z}$-value. Then we obtain for the total differential cross section $\sigma$ and for the corresponding spin-flip probability $Q$ :

$\sigma=\sum_{m=-I}^{I} g(m, T)\left[\frac{1}{2}\left(1+p_{0 z}\right) \sigma^{+}(m)+\frac{1}{2}\left[(u) \_\rho\left(1-p_{0 z}\right)\right.\right.$

$Q=\frac{\sigma_{f}}{\sigma}=\frac{1}{\sigma} \sum_{m=-1}^{I} g(m, T)\left[\frac{1}{2}\left(1+p_{0 z}\right) \sigma_{\mathrm{f}}^{+}(m)\right.$

where from Eqs. (22) - (29)

$$
\left.+\frac{1}{2}\left(1-p_{0 z}\right) \sigma_{\mathrm{f}}^{-}(m)\right]
$$

$$
\begin{aligned}
\sigma^{ \pm}(m)= & o_{\mathrm{nf}}^{ \pm}(m)+\sigma_{\mathrm{f}}^{ \pm}(m) \\
= & \sigma_{\mathrm{nf}}^{0 \pm}(m)+\sigma_{\mathrm{nf}}^{\prime \pm}(m)+\sigma_{\mathrm{nf}}^{\prime \prime \pm}(m)+\sigma_{\mathrm{f}}^{0 \pm}(m) \\
& +\sigma_{\mathrm{f}}^{\prime}(m)+\sigma_{\mathrm{nf}}^{\prime \prime}(m) .
\end{aligned}
$$

$$
\begin{aligned}
& \sigma_{\mathrm{nf}}=\left[(2 \pi)^{3} / V_{0}\right] \sum_{\tau} e^{-2 W} \cdot \frac{1}{2}\left(1+p_{0 z}\right)\left\{\left|\bar{f}_{\mathrm{nf}}^{0+}\right|^{2} \delta\left(\boldsymbol{k}_{\mathrm{e}}-\boldsymbol{k}_{0}-\boldsymbol{\tau}\right)+\left(k^{\prime} \mid k_{0}\right)\left|\bar{f}_{\mathrm{nf}}^{\prime+}\right|^{2} \delta\left(\boldsymbol{k}_{\mathrm{e}}-\boldsymbol{k}_{0}-\boldsymbol{\tau}\right)\right. \\
& \left.+\left(k^{\prime \prime} / k_{0}\right)\left|\bar{f}_{\mathrm{nf}}^{\prime \prime}\right|^{2} \delta\left(\boldsymbol{k}_{\mathrm{e}}-\boldsymbol{k}^{\prime \prime}-\boldsymbol{\tau}\right)\right\}+\left[(2 \pi)^{3} / V_{0}\right] \sum_{\boldsymbol{\tau}} e^{-2 W} \cdot\left(1-p_{0 z}\right)\left\{\left|\frac{1}{2} \bar{f}_{\mathrm{nf}}^{0-}\right|^{2} \delta\left(\boldsymbol{k}_{\mathrm{e}}-\boldsymbol{k}^{\prime \prime}-\boldsymbol{\tau}\right)\right. \\
& \left.+\left(k^{\prime} / k_{0}\right)\left|\bar{f}_{\mathrm{nf}}^{\prime}\right|^{2} \delta\left(\boldsymbol{k}_{\mathrm{e}}-\boldsymbol{k}^{\prime}-\boldsymbol{\tau}\right)+\left(k^{\prime \prime} / k_{0}\right) \mid \bar{f}_{\mathrm{nf}}^{\prime \prime}-2 \delta\left(\boldsymbol{k}_{\mathrm{e}}-\boldsymbol{k}^{\prime \prime}-\boldsymbol{\tau}\right)\right\}
\end{aligned}
$$

where $e^{-2 W}$ is the Debye-Waller factor described in Sect 2. and

$$
\bar{f}=\sum_{m=-1}^{l} f(m) g(m, \mathrm{~T}), \quad k^{\prime} / k_{0}=\operatorname{Re} \sqrt{1+2 m_{\mathrm{N}} b / \hbar^{2} k_{0}^{2}}, \quad k^{\prime \prime} / k_{0}=\operatorname{Re} \sqrt{1-2 m_{\mathrm{N}} b / \hbar^{2} k_{0}^{2}} .
$$

$V_{0}$ is the volume of the unit cell and $\tau$ is a reciprocal lattice vector multiplied by $2 \pi$. In Eq. (34) $f(m)$ is any one of Eqs. (16) - (21).

2 J. M. Blatt and L. C. Biedenharn, Phys. Rev. 86, 399 [1952] and 93, 1387 [1954].
3 L. Hulthén and M. Sugawara, Handbuch der Physik, Vol. 39, p. 83, Verlag Springer, Berlin 1957. 
In the same way we obtain

$$
\begin{aligned}
& \sigma_{\mathrm{f}}=\left[(2 \pi)^{3} / V_{0}\right] \sum_{\boldsymbol{\tau}} e^{-2 W} \cdot \frac{1}{2}\left(1+p_{0 z}\right)\left\{\left|\bar{f}_{\mathrm{f}}^{0+}\right| 2 \delta\left(\boldsymbol{k}_{\mathrm{e}}-\boldsymbol{k}_{0}-\boldsymbol{\tau}\right)+\left(k^{\prime} / k\right)\left|\bar{f}_{\mathrm{f}}^{\prime}+\right| 2 \delta\left(\boldsymbol{k}_{\mathrm{e}}-\boldsymbol{k}^{\prime}-\boldsymbol{\tau}\right)\right. \\
& \left.+\left(k^{\prime \prime} / k_{0}\right) \mid \bar{f}_{\mathrm{f}}^{\prime \prime}+2 \delta\left(\boldsymbol{k}_{\mathrm{e}}-\boldsymbol{k}^{\prime \prime}-\boldsymbol{\tau}\right)\right\}+\left[(2 \pi)^{3} / V_{0}\right] \sum_{\tau} e^{-2 W} \cdot \frac{1}{2}\left(1-p_{0 z}\right)\left\{\left|\bar{f}_{\mathrm{f}}^{0-}\right| 2\right\} \delta\left(\boldsymbol{k}_{\mathrm{e}}-\boldsymbol{k}_{\mathbf{0}}-\boldsymbol{\tau}\right) \\
& \left.+\left(\boldsymbol{k}^{\prime} / k\right)\left|\bar{f}_{\mathrm{f}}^{\prime}-\right| 2 \delta\left(\boldsymbol{k}_{\mathrm{e}}-\boldsymbol{k}^{\prime}-\boldsymbol{\tau}\right)+\left(k^{\prime \prime} / k_{0}\right)\left|\bar{f}_{\mathrm{f}}^{\prime \prime}-\right| 2 \delta\left(\boldsymbol{k}_{\mathrm{e}}-\boldsymbol{k}^{\prime \prime}-\boldsymbol{\tau}\right)\right\} .
\end{aligned}
$$

Using Eqs. (33) and (35) we obtain the corresponding spin-flip probability

$$
Q=\sigma_{\mathrm{f}} / \sigma \quad \text { with } \quad \sigma=\sigma_{\mathrm{nf}}+\sigma_{\mathrm{f}} .
$$

This $Q$ is a function of the observation direction and of the temperature, as we see from Eq. (1).

If we include only the first term of each bracket in Eq. (35) we obtain from Eq. (36) the probability of recoilless spin-flip scattering

$$
Q^{0}=\sigma_{\mathrm{f}}^{0} / \sigma=(1 / \sigma) \sum_{\tau}\left[(2 \pi)^{3} / 2 V_{0}\right] e^{-2 W}\left\{\left(1+p_{0 z}\right)\left|\tilde{f}_{\mathrm{f}}^{0+}\right| 2+\left(1-p_{0 z}\right)\left|\tilde{f}_{\mathrm{f}}^{0-}\right| 2\right\} \delta\left(\boldsymbol{k}_{\mathrm{e}}-\boldsymbol{k}_{0}-\boldsymbol{\tau}\right) .
$$

$Q^{0}$ contains only the elastic s.-o. processes which arise from the magnetic nuclear scattering.

\section{Magnetic Scattering by Oriented Atoms in Ferromagnetic Crystals}

\subsection{Conserved Quantities and Spin-Orbit Transitions}

In order to describe the scattering by the electronic shells of the magnetic atoms in a crystal we start from the Hamiltonian

$\mathcal{H}_{\mathrm{m}}=-\frac{\hbar^{2}}{2 m_{\mathrm{N}}} \nabla \frac{2}{\mathrm{~N}}+\sum_{j} H_{j}+2 \mu_{0} \gamma_{\mathrm{N}} \mu_{\mathrm{n}} \sum_{l}\left\{\frac{\left[\boldsymbol{s}_{l}\left(\boldsymbol{r}_{l}-\boldsymbol{r}_{\mathrm{N}}\right)\right]\left[\boldsymbol{\sigma}_{\mathrm{N}}\left(\boldsymbol{r}_{l}-\boldsymbol{r}_{\mathrm{N}}\right)\right]}{\left|\boldsymbol{r}_{l}-\boldsymbol{r}_{\mathrm{N}}\right|^{5}}-\frac{\boldsymbol{\sigma}_{\mathrm{N}} \boldsymbol{s}_{l}}{\left|\boldsymbol{r}_{l}-\boldsymbol{r}_{\mathrm{N}}\right|^{3}}+4 \pi \boldsymbol{\sigma}_{\mathrm{N}} \boldsymbol{s}_{l} \delta\left(\boldsymbol{r}_{l}-\boldsymbol{r}_{\mathrm{N}}\right)\right\}$

where $\boldsymbol{s}_{l}$ is the spin of the $l^{\text {th }}$ electron, $\boldsymbol{\sigma}_{\mathrm{N}}$ is the Pauli spin operator, and were again the subscript $\mathrm{N}$ distinguishes quantities characterizing the neutron. The index $j$ runs over the atoms and $l$ over all electrons of the crystal. $\mu_{0}$ is the Bohr-magneton and $H_{j}$ the Hamiltonian of $j^{\text {th }}$ atom. The interaction which appears in Eq. (38) is a sum of magnetic dipoledipole interactions of the magnetic moment of the neutron with the spins of the electrons. The interaction with the orbital magnetic momenta of the electrons has been omitted. This approximation is trivially good for atoms in an S state, fair for 3d atoms whose orbital ground state is a singlet and poor for most other cases (e. g. for rare earths). In the last case, for small scattering vector $q\left(q^{-1}>\right.$ atomic radius) we obtain a good approximation just replacing the spin of the atom $S_{j}$ in the matrix element (43) by $L_{j}+2 S_{j}$ which is proportional to its total magnetic moment.

As was the case for nuclear magnetic scattering it is easy to see that $s_{z}+S_{z}$ is not conserved

$$
\left[s_{z}+S_{z}, \mathcal{H}_{\mathrm{m}}\right] \neq 0 \text {; }
$$

4 O. Halpern and M. H. Johnson, Phys. Rev. 55, 898 [1939]. $s_{z}$ is the $z$-component of the neutron spin and $S_{z}$ that of the total electronic spin in the crystal. Denoting by $\mathcal{L}_{z}$ the $z$-component of the total electronic orbital agular momentum and by $L_{z}$ that of the neutron,

$$
\left[\mathcal{L}_{z}, \mathcal{H}_{\mathrm{m}}\right] \neq 0, \quad\left[L_{z}, \mathcal{H}_{\mathrm{m}}\right] \neq 0 .
$$

Only the total angular momentum is conserved

$$
\left[s_{z}+S_{z}+\mathcal{L}_{z}+L_{z}, \mathcal{H}_{\mathrm{m}}\right]=0 .
$$

From Eq. (38) one can derive the following form of the interaction in the momentum-representation (HALPERN and JOHNSON ${ }^{4}$ )

$$
\begin{aligned}
V_{\boldsymbol{P}^{\prime} \boldsymbol{P}}=\frac{4 \pi \hbar^{2}}{m_{\mathrm{N}}} r_{\mathbf{0}} \gamma_{\mathrm{N}} \sum_{j} \boldsymbol{F}_{j}(\boldsymbol{q}) \exp \left\{i \boldsymbol{q} \boldsymbol{R}_{j}\right\} \\
\cdot\left[\boldsymbol{S}_{j} \boldsymbol{s}-\left(\boldsymbol{e} \boldsymbol{S}_{j}\right)(\boldsymbol{e s})\right]
\end{aligned}
$$

where $r_{0}=e^{2} / m_{0} c^{2}$ is the electromagnetic radius of the electron, $\boldsymbol{R}_{j}$ is the position of the $j^{\text {th }}$ atom in the lattice, $\boldsymbol{q}=\boldsymbol{p}^{\prime}-\boldsymbol{p}$ is the scattering vector, $\boldsymbol{e}=\boldsymbol{q} / \boldsymbol{q}$ the corresponding unit vector, $\boldsymbol{S}_{j}$ the total electronic spin of the $j^{\text {th }}$ atom and

$$
F_{j}(\boldsymbol{q}) \equiv \int \Psi_{j}^{*} \sum_{\nu=1}^{Z_{j}} \frac{\exp \left\{i \boldsymbol{q} \boldsymbol{r}_{v}\right\} \boldsymbol{s}_{\nu} \mathbf{S}_{j}}{S_{j}\left(S_{j}+1\right)} \Psi_{j} \mathrm{~d} \tau_{j}
$$


the magnetic formfactor of the $j^{\text {th }}$ atom. $\Psi_{j}$ is the wavefunction of the $Z_{j}$ electrons.

In Eq. (43) the elementary scatterers are lattice points. We shall use the form (43) throughout this paper. In the case of very slow neutrons $\left(10^{-3} \mathrm{eV}\right.$ and below) the formfactor becomes a constant. It is then interesting to see what the conserved quantities are. If the lattice points are considered to be identical, the index $j$ of the formfactor can also be dropped. We obtain

$$
\begin{gathered}
{\left[s_{z}+S_{z}, \mathcal{H}_{\mathrm{m}}\right]_{\boldsymbol{p}^{\prime} \boldsymbol{P}}=-\left(4 \pi \hbar^{2} / m_{\mathrm{N}}\right) r_{0} \gamma_{\mathrm{N}} F \sum_{j} \exp \left\{i \boldsymbol{q} \boldsymbol{R}_{j}\right\}\left[-\left(e_{x} S_{j y}-e_{y} S_{j x}\right)(\boldsymbol{e} \boldsymbol{s})-\left(e_{x} s_{y}-e_{y} s_{x}\right)\left(\boldsymbol{e} \mathbf{S}_{j}\right)\right] ;} \\
{\left[L_{z}, \mathcal{H}_{\mathrm{m}}\right]_{\boldsymbol{p}^{\prime} \boldsymbol{P}}=\left(q_{y} \cdot \partial / \partial q_{x}-q_{x} \cdot \partial / \partial q_{y}\right) V_{\boldsymbol{p}^{\prime} \boldsymbol{p}}=-\left(4 \pi \hbar^{2} / m_{\mathrm{N}}\right) r_{0} \gamma_{\mathrm{N}} F \sum \exp \left\{i \boldsymbol{q} \boldsymbol{R}_{j}\right\} \cdot\left\{\left[\left(e_{x} S_{j y}-e_{y} S_{j x}\right)(\boldsymbol{e} \boldsymbol{s}\right.\right.} \\
\left.\left.+\left(e_{x} s_{y}-e_{y} s_{x}\right)\left(\boldsymbol{e} \mathbf{S}_{j}\right)\right]+i\left(X_{j} q_{y}-Y_{j} \boldsymbol{q}_{x}\right)\left[\mathbf{S}_{j} \boldsymbol{s}-\left(\boldsymbol{e} \boldsymbol{S}_{j}\right)(\boldsymbol{e} \boldsymbol{s})\right]\right\} \\
{\left[\mathcal{L}_{z}, \mathcal{H}_{\mathrm{m}}\right]_{\boldsymbol{p}^{\prime} \boldsymbol{P}}=\left(4 \pi \hbar^{2} / m_{\mathrm{N}}\right) r_{0} \gamma_{\mathrm{N}} i F \sum_{j} \exp \left\{i \boldsymbol{q} \boldsymbol{R}_{j}\right\}\left(X_{j} q_{y}-Y_{j} q_{x}\right)\left[\boldsymbol{S}_{j} \boldsymbol{s}-\left(\boldsymbol{e} \mathbf{S}_{j}\right)(\boldsymbol{e} \boldsymbol{s})\right]}
\end{gathered}
$$

We have denoted the sums over the spins and over the orbital momenta of the atoms by $S$ and $\mathcal{L}$; we put $R_{j x}=X_{j}$, etc. If we add Eqs. (45), (46) and (47), we obtain Eq. (42) again. We observe that now $s_{z}+S_{z}+L_{z}$ is nearly conserved. Indeed the nonconservation of this quantity is due only to a small geometrical effect. This effect is due to the angular momentum which corresponds to the transferred momentum $\boldsymbol{q}$, as referred to the center of inertia of the crystal. It means that the angular momentum of the crystal $\mathcal{L}$ is not perfectly separable from the relative orbital angular momentum $\boldsymbol{L}$ of the system crystal-neutron.

From Eq. (7) and (8) we see that s.-o. transitions will result from the term $\left(\boldsymbol{e} \boldsymbol{S}_{j}\right)(\boldsymbol{e} \boldsymbol{s})$ of the Hamiltonian. This results in elastic spin-flip scattering as in the case of nuclear magnetic scattering. The possible channels, as well as their angular dependence are exactly the same as for nuclear magnetic scattering.

In order to include the inelastic processes in which phonons and spin waves participate, we shall now apply the Van Hove time-dependent formalism ${ }^{5}$.

\subsection{Elastic and Inelastic Magnetic Scattering}

The general expression for the cross section in the Van Hove formalism is

$$
\frac{\mathrm{d}^{2} \sigma}{\mathrm{d} \Omega \mathrm{d} E_{p^{\prime}}}=\frac{m_{\mathrm{N}}^{2}}{(2 \pi)^{3} \hbar^{5}} \frac{p^{\prime}}{p} \int_{-\infty}^{\infty} \mathrm{d} t \exp \left\{(i / \hbar)\left(E_{p^{\prime}}-E_{p}\right) t\right\}\left\langle V_{\boldsymbol{P}^{\prime} \boldsymbol{P}}^{+}(0) V_{\boldsymbol{P}^{\prime} \boldsymbol{P}}(t)\right\rangle
$$

where $V_{p^{\prime} p}(t)$ is the interaction matrix element in the Heisenberg representation, $\mathrm{d} \Omega$ the element of solid angle and the averaging is done with the statistical operator including spin average if the polarization of the neutrons is not analysed. If we insert the matrix element (43) in Eq. (48), we obtain for the total magnetic scattering by the electronic shells of the atoms

$$
\begin{aligned}
& \frac{\mathrm{d}^{2} \sigma}{\mathrm{d} \Omega \mathrm{d} E_{p^{\prime}}}=\left(r_{0} \gamma_{\mathrm{N}}\right)^{2} \frac{p^{\prime}}{p} F^{2}(\boldsymbol{q}) \sum_{j j^{\prime}} \sum_{\alpha \beta}\left(\delta_{\alpha \beta}-e_{\alpha} e_{\beta}\right) \int_{-\infty}^{\infty} \frac{\exp \left\{(i / \hbar)\left(E_{p^{\prime}}-E_{p}\right) t\right\}}{2 \pi \hbar}\left\langle\exp \left\{-i \boldsymbol{q} \boldsymbol{R}_{j}(0)\right\}\right. \\
& \cdot \exp \left\{i \boldsymbol{q} \boldsymbol{R}_{j^{\prime}}(t)\right\rangle\left\langle S_{j}{ }^{\alpha}(0) S_{j^{\beta}}(t)\right\rangle \mathrm{d} t .
\end{aligned}
$$

Here we assumed that spin-lattice correlations are negligible so that the average which enters Eq. (48) is factorizable. If we neglect interactions between the spins, the integral in Eq. (49) yields

$$
I_{0}=\frac{1}{3} \exp \left\{i \boldsymbol{q}\left(\boldsymbol{R}_{j}-\boldsymbol{R}_{j^{\prime}}\right)\right\} S(S+1) \delta\left(E_{p^{\prime}}-E_{p}\right) \delta_{\alpha \beta} \delta_{j j^{\prime}}
$$

which corresponds to the paramagnetic case. If spin interactions are not neglected, we write the correlation functions which appear in Eq. (49) in the following way:

$$
\begin{aligned}
\left\langle\exp \left\{-i \boldsymbol{q} \boldsymbol{R}_{j}(0)\right\} \cdot \exp \left\{i \boldsymbol{q} \boldsymbol{R}_{j^{\prime}}(t)\right\}\right\rangle=\left\langle\exp \left\{-i \boldsymbol{q} \boldsymbol{R}_{j}(0)\right\} \cdot \exp \left\{i \boldsymbol{q} \boldsymbol{R}_{j^{\prime}}(\infty)\right\}\right\rangle \\
+\left[\left\langle\exp \left\{-i \boldsymbol{q} \boldsymbol{R}_{j}(0)\right\} \cdot \exp \left\{i \boldsymbol{q} \boldsymbol{R}_{j^{\prime}}(t)\right\}\right\rangle-\left\langle\exp \left\{-i \boldsymbol{q} \boldsymbol{R}_{j}(0)\right\} \cdot \exp \left\{i \boldsymbol{q} \boldsymbol{R}_{j^{\prime}}(\infty)\right\}\right\rangle\right],
\end{aligned}
$$

5 L. Van Hove, Phys. Rev. 95, 249, 1374 [1954]. 


$$
\left\langle S_{j}^{\alpha}(0) S_{j}^{, \beta}(t)\right\rangle=\left\langle S_{j}^{\alpha}(0) S_{j}^{, \beta}(\infty)\right\rangle+\left[\left\langle S_{j}^{\alpha}(0) S_{j}^{, \beta}(t)\right\rangle-\left\langle S_{j}^{\alpha}(0) S_{j}{ }^{\beta}(\infty)\right\rangle\right] .
$$

In Eqs. (51) and (52) the first terem yields the elastic part of the scattering. Indeed, there should not be any correlation at $t=\infty$; thus the correlation functions are factorizable for $t=\infty$. As they do not contain the time any more, one obtains an energy-conserving $\delta$-function from the integral in Eq. (49) characteristic of elastic processes.

Finally, we write the integral which occurs in Eq. (49) as follows:

$$
\begin{aligned}
& I=\left\langle\exp \left\{-i \boldsymbol{q} \boldsymbol{R}_{j}\right\}\right\rangle\left\langle\exp \left\{i \boldsymbol{q} \mathbf{R}_{j^{\prime}}\right\}\right\rangle\left\langle S_{j}{ }^{\alpha}\right\rangle\left\langle S_{j^{\prime}}{ }^{\beta}\right\rangle \delta\left(E_{p^{\prime}}-E_{p}\right) \\
& +\left\langle S_{j}{ }^{\alpha}\right\rangle\left\langle S_{j^{\prime}}{ }^{\beta}\right\rangle \frac{1}{2 \pi \hbar} \int_{\infty}^{-\infty}\left[\left\langle\exp \left\{-i \boldsymbol{q} \boldsymbol{R}_{j}(0)\right\} \cdot \exp \left\{i \boldsymbol{q} \boldsymbol{R}_{j^{\prime}}(t)\right\}\right\rangle-\left\langle\exp \left\{-i \boldsymbol{q} \boldsymbol{R}_{j}\right\}\right\rangle\left\langle\exp \left\{i \boldsymbol{q} \boldsymbol{R}_{j^{\prime}}\right\}\right\rangle\right] \mathrm{d} t \\
& +\left\langle\exp \left\{-i \boldsymbol{q} \boldsymbol{R}_{j}\right\}\right\rangle\left\langle\exp \left\{i \boldsymbol{q} \boldsymbol{R}_{j^{\prime}}\right\}\right\rangle \frac{1}{2 \pi \hbar} \int_{\infty}^{-\infty}\left[\left\langle S_{j}^{\alpha}(0) S_{j^{\prime}}{ }^{\beta}(t)\right\rangle-\left\langle S_{j}{ }^{\alpha}\right\rangle\left\langle S_{j}{ }^{\beta}\right\rangle\right] \mathrm{d} t+\ldots
\end{aligned}
$$

The term not included in Eq. (53) involves the inelastic scattering of neutrons by at least one phonon + one spin wave, i. e. multi-quanta processes not already included in (53). Multi-quanta processes are negligible at temperatures which are low compared to both the Debye and the Curie temperatures of the crystal.

\subsection{The Mössbauer-Effect and the Zero-Magnon Probability}

\section{a) The Mössbauer-Effect}

If we restrict ourselves to the first two terms of Eq. (53), decompose the position vectors $\boldsymbol{R}_{j}$ in mean value and fluctuation $\boldsymbol{R}_{j}=\boldsymbol{R}_{j}{ }^{0}+\boldsymbol{U}_{j}$ and express $\boldsymbol{U}_{j}$ in terms of phonon creation and annihilation operators, we obtain

$$
\begin{aligned}
I^{\prime}= & \exp \left\{i \boldsymbol{q}\left(\boldsymbol{R}_{j^{\prime}}{ }^{0}-\boldsymbol{R}_{j}{ }^{0}\right)\right\}\left\langle S_{j}{ }^{a}\right\rangle\left\langle S_{j^{\prime}}{ }^{\beta}\right\rangle \exp \left\{-2 W_{\boldsymbol{q}}\right\}\left\{\delta\left(E_{p^{\prime}}-E_{p}\right)+\sum_{\boldsymbol{k}, \varrho} \frac{\hbar\left[\boldsymbol{q} \boldsymbol{e}_{\varrho}(\boldsymbol{k})\right]^{2}}{2 M N \omega_{\varrho}(\boldsymbol{k})} \exp \left\{i \boldsymbol{k}\left(\boldsymbol{R}_{j^{0}}-\boldsymbol{R}_{j^{0}}{ }^{0}\right\}\right.\right. \\
& \cdot\left[1+n_{\ell}(\boldsymbol{k})\right] \delta\left(E_{p^{\prime}}-E_{p}+\hbar \omega_{\varrho}(\boldsymbol{k})\right)+\sum_{k, \varrho} \frac{\hbar\left[\boldsymbol{q} \boldsymbol{e}_{\varrho}(\boldsymbol{k})\right]^{2}}{2 M N \omega_{\varrho}(\boldsymbol{k})} \exp \left\{-i \boldsymbol{k}\left(\boldsymbol{R}_{j}{ }^{0}-\boldsymbol{R}_{j^{0}}{ }^{0}\right)\right\} n_{\ell}(\boldsymbol{k}) \\
& \left.\cdot \delta\left(E_{p^{\prime}}-E_{p}-\hbar \omega_{\varrho}(\boldsymbol{k})\right)+\ldots\right\}
\end{aligned}
$$

where the dots indicate multi-phonon terms. In Eq. (54) we put

$$
W_{\boldsymbol{q}} \equiv(\hbar / 2 M N) \sum_{\boldsymbol{k}, \varrho,}\left(\left[\boldsymbol{q} \boldsymbol{e}_{\ell}(\boldsymbol{k})\right]^{2} / \omega_{\ell}(\boldsymbol{k})\right)\left[n_{\ell}(\boldsymbol{k})+\frac{1}{2}\right]
$$

$\exp \left\{-2 W_{\boldsymbol{q}}\right\}$ is the Debye-Waller factor, $\boldsymbol{e}_{e}$ are the unit polarization vectors of the 3 acoustical phononbranches, $M, N$ the mass and the number of lattice atoms, $n_{\ell}(\boldsymbol{k})$ the Bose-expectation number of phonons at the temperature $T$. The first term in the curly brackets of Eq. (54) corresponds to elastic scattering, the second to the emission of one phonon and the third to the absorption of a phonon.

As we shall see, any average $\left\langle S_{j}{ }^{a}\right\rangle$ does not depend on $j$. The sum over $j$ and $j^{\prime}$ which is present in Eq. (11) can then be performed using

$$
\left|\sum_{j} \exp \left\{i \boldsymbol{n} \boldsymbol{R}_{j}{ }^{0}\right\}\right|^{2}=\left[(2 \pi)^{3} / V_{0}\right] N \sum_{\tau} \delta(\boldsymbol{n}-\boldsymbol{\tau})
$$

where $V_{0}$ is the volume of a unit cell, $N$ the total number of lattice points, $\sum_{\tau}$ a vector of the reciprocal lattice multiplied by $2 \pi$ and $\boldsymbol{n}$ an arbitrary vector. We obtain 


$$
\begin{aligned}
\sum_{j, j^{\prime}} I^{\prime}= & {\left[(2 \pi)^{3} / V_{0}\right] N\left\langle S^{x}\right\rangle\left\langle S^{\beta}\right\rangle \sum_{\tau} \exp \left\{-2 W_{\tau}\right\}\left\{\cdot \left\{\delta\left(E_{p^{\prime}}-E_{p}\right) \delta(\boldsymbol{q}-\boldsymbol{\tau})\right.\right.} \\
& +\sum_{\boldsymbol{k}, \varrho} \frac{\hbar\left[\boldsymbol{q} \boldsymbol{e}_{Q}(\boldsymbol{k})\right]^{2}}{2 M N \omega_{\varrho}(\boldsymbol{k})}\left[1+n_{\varrho}(\boldsymbol{k})\right] \delta\left(E_{p^{\prime}}-E_{p}+\hbar \omega_{\varrho}(\boldsymbol{k})\right) \delta(\boldsymbol{q}-\boldsymbol{k}-\boldsymbol{\tau}) \\
& \left.+\sum_{\boldsymbol{k}, \varrho} \frac{\hbar\left[\boldsymbol{q} \boldsymbol{e}_{\varrho}(\boldsymbol{k})\right]^{2}}{2 M N \omega_{\varrho}(\boldsymbol{k})} n_{\varrho}(\boldsymbol{k}) \delta\left(E_{p^{\prime}}-E_{p}-\hbar \omega_{\varrho}(\boldsymbol{k})\right) \delta(\boldsymbol{q}+\boldsymbol{k}-\boldsymbol{\tau})\right\} .
\end{aligned}
$$

In order to remove the $\delta$-functions, we integrate over $E_{p^{\prime}}$ and $\boldsymbol{p}^{\prime}$. In the last two terms of Eq. (57) $\boldsymbol{q}$ is replaced thus by $\boldsymbol{\tau} \pm \boldsymbol{k}$. The presence of $\boldsymbol{k}$ along with $\boldsymbol{\tau}$ does not introduce difficulties because $\omega_{Q}(\boldsymbol{k})$ $=\omega_{Q}(-\boldsymbol{k}), n_{Q}(\boldsymbol{k})=n_{Q}(-\boldsymbol{k})$ in the sums, and $\boldsymbol{e}_{Q} \boldsymbol{k}=0$ for the values of $\varrho$ which correspond to transversal phonons. In longitudinal phonon terms $k^{2}$ is negligible compared to $\tau^{2}$. Neglecting again multiphonon effects, $\exp \left\{-W_{\tau_{ \pm} k}\right\}$ can be replaced by $\exp \left\{-2 W_{\tau}\right\}$ in the last two terms of Eq. (57). Finally, we obtain

$$
\begin{aligned}
\left.\iint \sum_{j, j^{\prime}} I^{\prime} \mathrm{d} E_{p^{\prime}} \mathrm{d}^{3} p^{\prime}=\left[(2 \pi)^{3} / V_{0}\right] N\left\langle S^{\alpha}\right\rangle\left\langle S^{\beta}\right\rangle\right\} \sum_{\tau} \\
\cdot\left\{\exp \left\{-2 W_{\tau}\right\}+\exp \left\{-2 W_{\tau} \sum_{\boldsymbol{k}, \varrho} \frac{\hbar\left[\tau \boldsymbol{e}_{\varrho}(\boldsymbol{k})\right]^{2}}{2 M N \omega_{\varrho}(\boldsymbol{k})}\left[1+2 n_{\varrho}(\boldsymbol{k})\right]+\ldots\right\} .\right.
\end{aligned}
$$

The first term in curly brackets is the probability of recoilless (i. e. elastic) scattering. This also represents the probability for the Mössbauer-effect which is the recoilless emission of a $\gamma$-quantum of momentum q. It is essentially a function of the ratio of temperature to Debye-temperature for the given crystal. The second term is the probability of one-phonon exchange scattering (emission or absorption of a phonon). The points correspond to multi-phonon processes. The sum of all probabilities is 1 , as we can easily verify with the aid of Eq. (55) :

$$
\exp \left\{-2 W_{\tau}\right\}\left\{1+\sum_{\boldsymbol{k}, \varrho} \frac{\hbar\left[\tau \boldsymbol{e}_{Q}(\boldsymbol{k})\right]^{2}}{2 M N \omega_{Q}(\boldsymbol{k})}\left[1+2 n_{Q}(\boldsymbol{k})\right]+\ldots\right\}=\exp \left\{-2 W_{\tau}\right\} \exp \left\{2 W_{\tau}\right\}=1 .
$$

\section{b) The Zero-Magnon Probability}

Let us restrict our considerations to the first and third terms of Eq. (53). Then

$$
\begin{aligned}
I^{\prime \prime}=\exp \left\{i \boldsymbol{q}\left(\boldsymbol{R}_{j^{\prime}}{ }^{0}-\boldsymbol{R}_{j}{ }^{0}\right)\right\} \cdot \exp \left\{-2 W_{\boldsymbol{q}}\right\} \cdot\left\{\left\langle S_{j}{ }^{\alpha}\right\rangle\left\langle S_{j^{\beta}}{ }^{\beta}\right\rangle \delta\left(E_{p^{\prime}}-E_{p}\right)\right. & (60) \\
& \left.+\frac{1}{2 \pi \hbar} \int_{-\infty}^{\infty}\left[\left\langle S_{j}{ }^{\alpha}(0) S_{j^{\prime}}(t)\right\rangle-\left\langle S_{j}{ }^{\alpha}\right\rangle\left(S_{j^{\prime}}\right)\right] \mathrm{d} t\right\} .
\end{aligned}
$$

We start from the simple Heisenberg-model of a ferromagnet, with the Hamiltonian

$$
\mathcal{H}=-\sum_{j \neq j^{\prime}} \mathcal{J}\left(\boldsymbol{R}_{j}-\boldsymbol{R}_{j^{\prime}}\right)\left(S_{j} S_{j^{\prime}}\right)-2 \mu H \sum_{j} S_{j}^{z} .
$$

At low temperatures, the spin components can be expressed in terms of magnon creation and annihilation operators with the aid of the Holstein-Primakoff transformation

$$
S_{j}^{+} \cong \sqrt{2 S} b_{j}, S_{j}^{-} \cong \sqrt{2 S} b_{j}{ }^{+}, S_{j}{ }^{2} \cong S-b_{j}{ }^{+} b_{j}
$$

where $S_{j}^{ \pm}=S_{j}^{x} \pm S_{j}^{y}$. If we pass from the Bose creation and annihilation operators $b_{j}^{+}$and $b_{j}$ to their Fourier components

$$
b_{j}=\frac{1}{\sqrt{N}} \sum_{\boldsymbol{k}} \exp \left\{i \boldsymbol{k} \boldsymbol{R}_{j}{ }^{0}\right\} \cdot b_{\boldsymbol{k}}, \quad b_{j}^{+}=\frac{1}{\sqrt{N}} \sum \exp \left\{-i \boldsymbol{k} \boldsymbol{R}_{j}{ }^{0}\right\} \cdot b_{\boldsymbol{k}}^{+},
$$

the Hamiltonian is diagonalized up to second order in the $b$ 's

$$
\mathcal{H}=\sum_{\boldsymbol{k}} \varepsilon_{\boldsymbol{k}} b_{\boldsymbol{k}} ; \varepsilon_{\boldsymbol{k}}=2 S \sum_{\boldsymbol{R}} J(\boldsymbol{R})(1-\exp \{i \boldsymbol{k} \boldsymbol{R}\})+2 \mu H .
$$


In the case of a cubic (simple, face centered or body-centered) crystal with a lattice-constant $a$ we get

$$
\varepsilon_{\boldsymbol{k}}=2 S J_{0} a^{2} k^{2}+2 \mu H \text {. }
$$

Here $J_{0}$ is the interaction integral $J(\boldsymbol{R})$ for next neighbors, $\mu \boldsymbol{S}$ the magnetic moment of the electronic shell of the atom, $\boldsymbol{S}$ its spin and $H$ a static magnetic field.

If we include the factor $\delta_{\alpha \beta}-e_{\alpha} e_{\beta}$ of Eq. (49) we obtain

$$
\begin{aligned}
& \sum_{\alpha \beta}\left(\delta_{\alpha \beta}-e_{a} e_{\beta}\right)\left\langle S_{j}^{\alpha}(0) S_{j^{\prime}}{ }^{\beta}(t)\right\rangle=\frac{1}{2}\left\langle S_{j}{ }^{+} S_{j^{\prime}}{ }^{-}+S_{j}^{-} S_{j^{\prime}}{ }^{+}\right\rangle+\left\langle S_{j}{ }^{z} S_{j^{\prime}}{ }^{\tilde{*}}\right\rangle \\
& -\left\langle\left[\frac{1}{2}\left(S_{j}^{+} e^{-}+S_{j}^{-} e^{+}\right)+S_{j}^{z} e_{\mathrm{z}}\right]\left[\frac{1}{2}\left(S_{j^{\prime}}{ }^{+} e^{-}+S_{j^{\prime}} e^{+}\right)+S_{j^{\prime}} e_{z}\right]\right\rangle
\end{aligned}
$$

$=\frac{1}{4}\left\langle\left[S_{j}^{+}(0) S_{j^{\prime}}{ }^{-}(t)+S_{j}^{-}(0) S_{j^{\prime}}{ }^{+}(t)\right]\right\rangle\left[1+(\boldsymbol{e} \boldsymbol{m})^{2}\right]+\left\langle S_{j}^{z}(0) S_{j^{\prime}}{ }^{z}(t)\right\rangle\left[1-(\boldsymbol{e} \boldsymbol{m})^{2}\right]$

$=\frac{S}{2 N} \sum_{\boldsymbol{k}, \boldsymbol{k}}\left[\exp \left\{i\left(\boldsymbol{k} \boldsymbol{R}_{j}{ }^{0}-\boldsymbol{k}^{\prime} \boldsymbol{R}_{j^{\prime}}{ }^{0}\right)\right\} \cdot\left\langle b_{\boldsymbol{k}}(0) b_{\boldsymbol{k}^{\prime}}^{+}(t)\right\rangle\right.$

$$
\left.+\exp \left\{-i\left(\boldsymbol{k} \boldsymbol{R}_{j}^{0}-\boldsymbol{k}^{\prime} \boldsymbol{R}_{j^{0}}{ }^{0}\right)\right\} \cdot\left\langle b_{\boldsymbol{k}}^{+}(0) b_{\boldsymbol{k}^{\prime}}(t)\right\rangle\right]\left[1+(\boldsymbol{e} \boldsymbol{m})^{2}\right]
$$

$+\left\langle\left[S-\frac{1}{N} \sum_{\boldsymbol{k}, \boldsymbol{k}^{\prime}} \exp \left\{-i \boldsymbol{k} \boldsymbol{R}_{j}^{0}\right\} \cdot \exp \left\{i \boldsymbol{k}^{\prime} \boldsymbol{R}_{j}^{0}\right\} \cdot b_{\boldsymbol{k}}^{+}(0) b_{\boldsymbol{k}^{\prime}}(0)\right]\left[S-\frac{1}{N} \sum_{\boldsymbol{k}_{1}, \boldsymbol{k}_{\mathbf{k}^{\prime}}} \exp \left\{-i \boldsymbol{k}_{1} \boldsymbol{R}_{j^{\prime}}{ }^{0}\right\}\right.\right.$

$$
\left.\cdot \exp \left\{i \boldsymbol{k}_{1}^{\prime} \boldsymbol{R}_{j^{\prime}}{ }^{0}\right\} b_{\boldsymbol{k}_{1}}^{+}(t) b_{\boldsymbol{k}_{1}}(t)\right]>\left[1-(\boldsymbol{e} \boldsymbol{m})^{2}\right] .
$$

We denoted by $\boldsymbol{m}$ the unit vector of magnetization which is supposed to be in $z$-direction. The non-diagonal correlations $\left\langle S_{j}^{ \pm}(0) S_{j^{\prime}}(t)\right\rangle,\left\langle S_{j}^{ \pm}(0) S_{j^{\prime}}^{ \pm}(t)\right\rangle$ and $\left\langle S_{j}^{z}(0) S_{j^{\prime}}^{ \pm}(t)\right\rangle$ were neglected in Eq. (66) because they were comparatively small at low temperatures. In Eq. (27)

$$
\left\langle b_{\boldsymbol{k}}^{+}(0) b_{\boldsymbol{k}^{\prime}}(0)\right\rangle=\left\langle\boldsymbol{b}_{\boldsymbol{k}}^{+}(t) b_{\boldsymbol{k}^{\prime}}(t)\right\rangle=\langle n(\boldsymbol{k})\rangle \delta_{\boldsymbol{k} \boldsymbol{k}^{\prime}}=\left(\exp \left\{\beta \varepsilon_{\boldsymbol{k}}\right\}-1\right)^{-1} \delta_{\boldsymbol{k} \boldsymbol{k}^{\prime}}
$$

is the Bose-statistical expectation value of the magnon-number in equilibrium at the temperature $1 / \beta$. According to Eq. (64)

$$
\begin{aligned}
& \frac{1}{2 \pi \hbar} \int_{-\infty}^{\infty} \exp \left\{(i / \hbar)\left(E_{p^{\prime}}-E_{p}\right) t\right\} \cdot\left\langle b_{\boldsymbol{k}}(0) b_{\boldsymbol{k}}^{+}(t)\right\rangle \mathrm{d} t=[1+n(\boldsymbol{k})] \delta\left(E_{p^{\prime}}-E_{p}+\varepsilon_{\boldsymbol{k}}\right), \\
& \frac{1}{2 \pi \hbar} \int_{-\infty}^{\infty} \exp \left\{(i / \hbar)\left(E_{p^{\prime}}-E_{p}\right) t\right\} \cdot\left\langle b_{\boldsymbol{k}}^{+}(0) b_{\boldsymbol{k}}(t)\right\rangle \mathrm{d} t=n(\boldsymbol{k}) .
\end{aligned}
$$

Thus we obtain from Eq. (67)

$$
\begin{gathered}
\frac{1}{2 \pi \hbar} \int_{-\infty}^{\infty} \sum_{\alpha \beta}\left(\delta_{\alpha \beta}-e_{\alpha} e_{\beta}\right) \exp \left\{(i / \hbar)\left(E_{p^{\prime}}-E_{p}\right) t\right\}\left\langle S_{j}^{\alpha}(0) S j^{\beta}(t)\right\rangle \mathrm{d} t=\sum_{\boldsymbol{k}} \exp \left\{i \boldsymbol{k}\left(\boldsymbol{R}_{j}{ }^{0}-\boldsymbol{R}_{j^{\prime}}{ }^{0}\right)\right\} \\
\cdot\left[1+(\boldsymbol{e} \boldsymbol{m})^{2}\right](S / 2 N)\left[(1+n(\boldsymbol{k})) \delta\left(E_{p^{\prime}}-E_{p}+\varepsilon_{\boldsymbol{k}}\right)+n(\boldsymbol{k}) \delta\left(E_{p^{\prime}}-E_{p}-\varepsilon_{\boldsymbol{k}}\right)\right] \\
+\left[S^{2}-(2 S / N) \sum_{\boldsymbol{k}} n(\boldsymbol{k})\right] \delta\left(E_{p^{\prime}}-E_{p}\right)\left[1-(\boldsymbol{e} \boldsymbol{m})^{2}\right] .
\end{gathered}
$$

From Eqs. (62) and (63) one gets

$$
\left\langle S_{j}{ }^{\alpha}\right\rangle=\delta_{a 3}\left\langle S_{j}{ }^{z}\right\rangle=\delta_{a 3}\left[S-(1 / N) \sum_{\boldsymbol{k}} n(\boldsymbol{k})\right] \equiv \delta_{a 3} S(T) .
$$

We see that the mean values of the spin-components perpendicular to the direction of magnetization vanish. The mean value in the direction of magnetization is denoted by $S(T)$. Introducing Eqs. (71) and (72) into Eq. (60), we obtain

$$
\begin{aligned}
& \left.\sum_{\alpha \beta}\left(\delta_{\alpha \beta}-e_{\alpha} e_{\beta}\right)\right] I^{\prime \prime}=\exp \left\{i \boldsymbol{q}\left(\boldsymbol{R}_{j^{\prime}}{ }^{0}-\boldsymbol{R}_{j}{ }^{0}\right)\right\} \exp \left\{-2 W_{\boldsymbol{q}}\right\} \cdot\left\{S^{2}(T)\left[1-(\boldsymbol{e} \boldsymbol{m})^{2}\right] \delta\left(E_{p^{\prime}}-E_{p}\right)\right. \\
& \left.+\sum_{\boldsymbol{k}} \exp \left\{i \boldsymbol{k}\left(\boldsymbol{R}_{j}{ }^{\mathbf{0}}-\boldsymbol{R}_{j^{\prime}}\right)\right\} \cdot\left[1+(\boldsymbol{e} \boldsymbol{m})^{2}\right](S / 2 N)\left[(1+n(\boldsymbol{k})) \delta\left(E_{p^{\prime}}-E_{p}\right)+n(\boldsymbol{k}) \delta\left(E_{p^{\prime}}-E_{p}-\varepsilon_{\boldsymbol{k}}\right)\right]\right\} .
\end{aligned}
$$


From the arguments of the $\delta$-functions we see that the first term in curly brackets corresponds to elastic scattering and the second to the inelastic one-quantum scattering. The same was true for Eq. (60) already. Summing over $j$ and $j^{\prime}$ we obtain, analogous to $\mathrm{Eq}$. (57)

$\sum_{i j^{\prime}} \sum_{\alpha \beta}\left(\delta_{a \beta}-e_{a} e_{\beta}\right) I^{\prime \prime}=\left[(2 \pi)^{3} / V_{0}\right] N \sum_{\boldsymbol{\tau}} \exp \left\{-2 W_{\boldsymbol{q}}\right\} \cdot\left\{S^{2}(T)\left[1-(\boldsymbol{e} \boldsymbol{m})^{2}\right] \delta(\boldsymbol{q}-\boldsymbol{\tau}) \delta\left(E_{p^{\prime}}-E_{p}\right)\right.$

$\left.+\sum_{\boldsymbol{k}}\left[1+(\boldsymbol{e} \boldsymbol{m})^{2}\right](S / 2 N)\left[(1+n(\boldsymbol{k})) \delta(\boldsymbol{q}-\boldsymbol{k}-\boldsymbol{\tau}) \delta\left(E_{p^{\prime}}-E_{p}+\varepsilon_{\boldsymbol{k}}\right)+n(\boldsymbol{k}) \delta(\boldsymbol{q}+\boldsymbol{k}-\boldsymbol{\tau}) \delta\left(E_{p^{\prime}}-E_{p}-\varepsilon_{\boldsymbol{k}}\right)\right]\right\}$

As in Eq. (58) we shall again integrate over $E_{p^{\prime}}$ and $\boldsymbol{p}^{\prime}$ thus removing the $\delta$-functions. In fact we are not interested here in a direction-analysis of the outgoing neutrons. It is rather the mean probability for elastic and inelastic processes which we are interested in. Correspondingly, in Eq. (74) we average over the directions of $\boldsymbol{k}$ and $\tau$ which are subject to summations. We get $\frac{1}{3}$ for the average of $(\boldsymbol{e} \boldsymbol{m})^{2}$. We arrive finally at

$$
\begin{aligned}
& \iint \sum_{j j^{\prime}} \sum_{\alpha \beta}\left(\delta_{\alpha \beta}-e_{\alpha} e_{\beta}\right) I^{\prime \prime} \mathrm{d} E_{p^{\prime}} \mathrm{d}^{3} p^{\prime}=\frac{(2 \pi)^{3}}{V_{0}} N \sum_{\boldsymbol{\tau}} \exp \{-2 W \tau\} \frac{2}{3} S(S+1)(75) \\
& \cdot\left\{\frac{S^{2}(T)}{S(S+1)}+\frac{1}{N(S+1)} \sum_{\boldsymbol{k}}[1+2 n(\boldsymbol{k})]\right\} .
\end{aligned}
$$

The first term in the curly brackets is here the zero magnon probability which we shall denote by $\varkappa$. $\varkappa$ corresponds to the Debye-Waller factor which occurs in the Mössbauer-effect and in the elastic nuclear scattering of neutrons (58). The second term is the probability for emission or absorption of a spin-wave in the scattering process. The sum of these probabilities yields again 1 . This is obvious if we insert again the expression (72) for $S^{2}(T)$ and take into account the fact that the sum over $\boldsymbol{k}$ contains just $N$ terms

$$
[1 / S(S+1)]\left[S^{2}-(2 S / N) \sum_{\boldsymbol{k}} n(\boldsymbol{k})\right]+[1 /(S+1)]\left[1+(2 / N) \sum_{\boldsymbol{k}} n(\boldsymbol{k})\right]=1 .
$$

We observe that $\exp \left\{-2 W_{\boldsymbol{q}}\right\}$ still appears in Eqs. (73)-(75). It expresses the probability that no phonons will be emitted or absorbed in the process. In the same way the factor $\left.\left\langle S_{j}{ }^{a}\right\rangle\left\langle S_{j^{\prime}}\right\rangle\right\rangle=\delta_{a 3} \delta_{\beta 3} S^{2}(T)$ in Eq. (58) is necessary to ascertain that no spin-waves are changed in the process.

If we include multi-magnon scattering in our considerations taking further terms of (62) and (63) into account in Eq. (67), supplementary terms will appear in the curly brackets of Eq. (75). They correspond to additional terms of higher order which also appear in the expression for $S^{2}(T)$. Thus, they will again cancel and the normalization of probabilities (76) will still be valid.

If we restrict ourselves to spin-flip scattering only, the zero-magnon probability will be different from $\varkappa=S^{2}(T) / S(S+1)$ which is relative to the total scattering. We obtain the zero-magnon probability for spin-flip scattering, $\varkappa_{\mathrm{f}}$, by multiplying with the spin-flip probability for elastic scattering $Q^{0}$ from Eq. (85) and dividing by the total spin-flip probability $Q$ [Eq. (95)]:

$$
\varkappa_{\mathrm{f}}=\left(Q^{0} / Q\right) \varkappa .
$$

\subsection{Cross Sections of the Elastic and Inelastic Magnetic Scattering}

If we replace the integral which occurs in Eq. (49) by Eq. (15) we obtain, with the aid of Eqs. (57) and (74), the complete differential cross section for magnetic scattering, per atom

$$
\begin{gathered}
\frac{\mathrm{d} \sigma}{\mathrm{d} \Omega \mathrm{d} E_{p^{\prime}}}=\left(r_{0} \gamma_{\mathrm{N}}\right)^{2} F^{2}(\boldsymbol{q}) \exp \left\{-2 W_{\boldsymbol{q}}\right\} \frac{(2 \pi)^{3}}{V_{0}} \sum_{\tau}\left\{S^{2}(T)\left[1-(\boldsymbol{e} \boldsymbol{m})^{2}\right] \delta(\boldsymbol{q}-\boldsymbol{\tau}) \delta\left(E_{p^{\prime}}-E_{p}\right)\right. \\
+\frac{p^{\prime}}{p} \frac{S}{2 N}\left[1+(\boldsymbol{e} \boldsymbol{m})^{2}\right] \sum_{\boldsymbol{k}}\left[(1+n(\boldsymbol{k})) \delta(\boldsymbol{q}-\boldsymbol{k}-\boldsymbol{\tau}) \delta\left(E_{p^{\prime}}-E_{p}+\varepsilon_{\boldsymbol{k}}\right)+n(\boldsymbol{k}) \delta(\boldsymbol{q}+\boldsymbol{k}-\boldsymbol{\tau}) \delta\left(E_{p^{\prime}}-E_{p}-\varepsilon(\boldsymbol{k})\right)\right] \\
+\frac{p^{\prime}}{p} S^{2}(T)\left[1-(\boldsymbol{e} \boldsymbol{m})^{2}\right] \sum_{\boldsymbol{k}} \frac{\hbar\left[\boldsymbol{q} \boldsymbol{e}_{Q}(\boldsymbol{k})\right]^{2}}{2 M N \omega_{\ell}(\boldsymbol{k})}\left[(1+n(\boldsymbol{k})) \delta(\boldsymbol{q}-\boldsymbol{k}-\boldsymbol{\tau}) \delta\left(E_{p^{\prime}}-E_{p}+\hbar \omega_{Q}(\boldsymbol{k})\right)\right. \\
\left.\left.+n(\boldsymbol{k}) \delta(\boldsymbol{q}+\boldsymbol{k}-\boldsymbol{\tau}) \delta\left(E_{p^{\prime}}-E_{p}-\hbar \omega_{Q}(\boldsymbol{k})\right)\right]\right\}
\end{gathered}
$$


The first term corresponds to elastic scattering. The second describes the scattering with emission of a magnon [the part with $1+n(\boldsymbol{k})$ ] and with absorption of a magnon [the part proportional to $n(\boldsymbol{k})$ ]. In the same way, the last term represents magnetic scattering with emission or absorption of a phonon (magnetovibrational scattering).

\subsection{Spin-Flip in the Elastic Scattering}

In order to include the interference of magnetic scattering with nuclear scattering we shall treat the problem of elastic scattering of polarized neutrons starting from the total (nuclear, nuclear magnetic and magnetic) scattering amplitude. We have to insert into Eq. (48)

$$
V_{p^{\prime} p}=\sum_{j}\left\{c_{j}+\frac{1}{2} d_{j} \mathbf{I} \boldsymbol{\sigma}+2 \pi \varepsilon[\mathbf{I} \boldsymbol{\sigma}-(\mathbf{I} \boldsymbol{e})(\boldsymbol{\sigma} \boldsymbol{e})]-\frac{2 \pi \hbar^{2}}{m_{\mathrm{N}}} r_{0} \gamma_{\mathrm{N}} F_{j}(\boldsymbol{q})\left[\mathbf{S}_{j} \boldsymbol{\sigma}-\left(\boldsymbol{e} \mathbf{S}_{j}\right)(\boldsymbol{e} \boldsymbol{\sigma})\right]\right\} \exp \left\{i \boldsymbol{q} \boldsymbol{R}_{j}\right\} .
$$

$\boldsymbol{\sigma}$ is the Pauli spin operator of the neutron. The third term in the curly brackets is the matrix element of the last term of Eq. (2). The polarization of the incident neutrons is given by the polarizaton vector $\boldsymbol{p}_{0}$ which is twice their average spin. This corresponds to the spin density matrix

$$
\varrho=\frac{1}{2}\left(1+\boldsymbol{p}_{0} \boldsymbol{\sigma}\right) ;\langle\boldsymbol{s}\rangle=\frac{1}{2}\langle\boldsymbol{\sigma}\rangle=\frac{1}{2} \operatorname{Tr} \frac{1}{2} \boldsymbol{\sigma}\left(1+\boldsymbol{p}_{0} \boldsymbol{\sigma}\right)=\frac{1}{2} \boldsymbol{p}_{0} .
$$

$p_{0 z}$ was previously introduced in Sect. 1.3 .

Inserting Eq. (79) in Eq. (48) and calculating the trace with $\varrho$ we obtain the differential cross section.

$$
\sigma^{\mathbf{0}}=\left[A^{2}+B^{2} M^{2}+2 A B\left(\boldsymbol{M} \mathbf{p}_{0}\right)\right] \frac{(2 \pi)^{3}}{V_{0}} \exp \left\{-2 W_{\mathbf{q}}\right\} \sum_{\boldsymbol{\tau}} \delta(\boldsymbol{q}-\boldsymbol{\tau})
$$

for the elastic coherent scattering of neutrons with the initial polarization $p_{0}$. We have used the well known expressions for the traces of products of Pauli matrices and we introduced the notations

$$
\begin{array}{rlrl}
A & \equiv\left(m_{\mathrm{N}} / 2 \pi \hbar^{2}\right)\left[c+\frac{1}{2} \bar{m} d_{0}\left(\boldsymbol{m} \boldsymbol{p}_{0}\right)\right] ; & & \boldsymbol{M} \equiv \boldsymbol{m}-(\boldsymbol{e} \boldsymbol{m}) \boldsymbol{e}, \\
B & \equiv\left(m_{\mathrm{N}} / \hbar^{2}\right) \varepsilon \bar{m}-r_{0} \gamma_{\mathrm{N}} F(\boldsymbol{q}) S(T) ; & \bar{m} \equiv \sum_{m=-l}^{I} m g(m, T) .
\end{array}
$$

Here $A$ is the coherent nuclear scattering amplitude, $B$ is the magnetic and nuclear-magnetic scattering amplitude and $g(m, T)$ is defined by Eq. (1). The last term in the square brackets of Eq. (81) describes the interference of nuclear and magnetic scattering. Except for nuclear magnetic scattering, Eq. (81) corresponds to previous calculations ${ }^{6,7}$.

The polarization of the scattered neutrons is twice the mean value of the spin

$$
\boldsymbol{p}=\frac{\operatorname{Tr}\left[\varrho\left\langle V_{p^{\prime} p}^{+} \sigma V_{p^{\prime} p}\right\rangle\right]}{\operatorname{Tr}\left[\varrho\left\langle V_{p^{\prime} p}^{+} V_{p^{\prime} p}\right\rangle\right]} .
$$

If we calculate the traces we obtain 7,8

$$
p=\frac{A^{2} \boldsymbol{p}_{0}+B^{2}\left[2 \boldsymbol{M}\left(\boldsymbol{M} \boldsymbol{p}_{0}\right)-M^{2} \boldsymbol{p}_{0}\right]+2 A B \boldsymbol{M}}{A^{2}+B^{2} M^{2}+2 A B\left(\boldsymbol{M} \boldsymbol{p}_{0}\right)} .
$$

Let us analyse the scattered neutrons in the direction of polarization of the incident neutrons $\boldsymbol{p}_{0}$. In order to calculate the spin-flip probability for elastic scattering $Q^{0}$, we consider a completely polarized incident beam $\left(\left|\boldsymbol{p}_{0}\right|=1\right) . Q^{0}$ is then given by the difference of the final and initial polarizations

$$
Q^{0}=\frac{1}{2}\left(1-\boldsymbol{p} \boldsymbol{p}_{0}\right)_{p_{0}=1}=\frac{B^{2}\left[M^{2}-\left(\boldsymbol{M} \boldsymbol{p}_{0}\right)^{2}\right]}{A^{2}+B^{2} M^{2}+2 A B\left(\boldsymbol{M} \boldsymbol{p}_{0}\right)} .
$$

6 A. W. Saenz, Phys. Rev. 119, 1542 [1960].

7 Yu. A. Izyumov, Usp. Fiz. Nauk 80, 41 [1963]; engl transl. Soviet Phys.-Usp. 16, 359 [1963].
8 Yu. A. Izyumov and S. V. Maleev, Zh. Eksperim. Teor. Fiz. 41, 1644 [1961] ; engl. transl. Soviet Phys.-JETP 14, 1168 [1962]. 
If we multiply Eq. (81) by $Q_{0}$, we obtain the elastic spin-flip cross section

$$
\sigma_{\mathrm{f}}^{0}=B^{2}\left[M^{2}-\left(\boldsymbol{M} \boldsymbol{p}_{0}\right)^{2}\right] \frac{(2 \pi)^{3}}{V_{0}} \exp \left\{-2 W_{\boldsymbol{q}}\right\} \cdot \sum_{\tau} \delta(\boldsymbol{q}-\tau) .
$$

The corresponding non-flip cross section will be

$$
\sigma_{\mathrm{nf}}^{0}=\left[A+B \boldsymbol{M} \boldsymbol{p}_{0}\right]^{2} \frac{(2 \pi)^{3}}{V_{0}} \exp \left\{-2 W_{\boldsymbol{q}}\right\} \cdot \sum_{\boldsymbol{\tau}} \delta(\boldsymbol{q}-\boldsymbol{\tau}) .
$$

If we add Eq. (86) and (87), Eq. (81) results: $\sigma^{0}=\sigma_{\mathrm{nf}}^{0}+\sigma_{\mathrm{f}}^{0} ; Q=\sigma_{\mathrm{f}} / \sigma$. Note that changing $\boldsymbol{p}_{0}$ to $-\boldsymbol{p}_{0}$ does not change the spin-flip scattering, whereas $\sigma_{\text {nf }}$ is changed by the interference term. This is just the term which yields a polarization of the scattered neutrons if unpolarized neutrons interact with a ferromagnet. This is observed in Eq. (84) too. The resulting polarization lies in the direction of the magnetization-component which is perpendicular to $\boldsymbol{q}$. It is produced by the non-flip scattering alone.

If $\boldsymbol{p}_{0}= \pm \boldsymbol{m}$ one gets the cross sections $\sigma_{\mathrm{f}}^{0 \pm}$ and $\sigma_{\mathrm{nf}}^{0+}$ from Eqs. (86) and (87). The angular dependence is the same as in Eqs. (23), (22), (35) and (33)

$$
M^{2}-\left(M p_{0}\right)^{2}=\frac{1}{4} \sin ^{2} 2 \theta_{0} ; \quad\left(M p_{0}\right)^{2}=\sin ^{4} \theta_{0} \quad\left(p_{0}= \pm m, \cos \theta_{0}=m e\right) .
$$

The elastic spin-flip scattering described by Eq. (86) corresponds to transitions in which $\Delta L=2, \Delta L_{z}= \pm 1$. These are spin-orbit transitions, as defined in Sect. 1.1, which result in spin-flip without spin-recoil. Instead of scattering by a nucleus, we are dealing here with scattering by the whole crystal, although the cross section is devided by $N$. The essential point is that spin-flip processes occur in which angular momentum is transferred to the crystal as a whole.

\subsection{Inelastic Spin-Flip Scattering}

The elastic spin-flip scattering which was treated in the last section is dominant at low temperatures (compared with the Curie temperature). At higher temperatures inelastic spin-flip processes are important also. These do not contain interference terms because the energies and wave vectors of neutrons magnetically scattered in various inelastic channels are different. These wave vectors are not equal to those of the neutrons which are subject to nuclear scattering. We shall insert again the amplitude (79) in Eq. (48). This time, however, we restrict ourselves to the last term of Eq. (53), which describes scattering with emission or absorption of spin-waves. For neutrons with the initial polarization $\boldsymbol{p}_{\mathbf{0}}$, the differential cross section per atom, for one-magnon scattering is

$$
\begin{aligned}
\sigma^{\prime}, " & =\left(r_{0} \gamma_{N}\right)^{2} F^{2}(\boldsymbol{q}) \frac{1}{2} S \exp \left\{-2 W_{\boldsymbol{q}}\right\} \cdot\left[1+(\boldsymbol{e} \boldsymbol{m})^{2} \mp 2(\boldsymbol{e} \boldsymbol{m})\left(\boldsymbol{e} \boldsymbol{p}_{0}\right)\right] \\
& \cdot \sum_{\boldsymbol{\tau}} \int \mathrm{d}^{3} k\left(p^{\prime} / p\right)\left[n(\boldsymbol{k})+\frac{1}{2} \pm \frac{1}{2}\right] \delta(\boldsymbol{q} \mp \boldsymbol{k}-\boldsymbol{\tau}) \delta\left(E_{p^{\prime}}-E_{p} \pm \varepsilon_{\boldsymbol{k}}\right) .
\end{aligned}
$$

The upper signs in this formula refer to scattering with emission of a spin-wave $\left(\sigma^{\prime}\right)$ and the lower to scattering with absorption $\left(\sigma^{\prime \prime}\right)$. The polarization of the inelastically scattered neutrons is determined ${ }^{8}$ from Eq. (83) to be

$$
\boldsymbol{p}^{\prime}, "=\frac{ \pm 2(\boldsymbol{e} \boldsymbol{m}) \boldsymbol{e}+2 \boldsymbol{X}\left(\boldsymbol{X} \boldsymbol{p}_{0}\right)+2 \mathbf{Y}\left(\mathbf{Y} \boldsymbol{p}_{0}\right)-\left(X^{2}+Y^{2}\right) \boldsymbol{p}_{0}}{1+(\boldsymbol{e} \boldsymbol{m})^{2} \mp 2(\boldsymbol{e} \boldsymbol{m})\left(\boldsymbol{e} \boldsymbol{p}_{0}\right)}
$$

We have chosen the $z$-axis in the direction of $\boldsymbol{m}$ and put $\boldsymbol{X}=\boldsymbol{u}_{x}+\left(\boldsymbol{e} \boldsymbol{u}_{z}\right) \boldsymbol{e}, \mathbf{Y}=\boldsymbol{u}_{y}+\left(\boldsymbol{e} \boldsymbol{u}_{y}\right) \boldsymbol{e} . \boldsymbol{u}_{x}$ and $\boldsymbol{u}_{y}$ are the unit vectors in the $x$ and $y$ directions.

From Eq. (90) we obtain the spin-flip probabilities $Q^{\prime}, "$

$$
Q^{\prime, "}=\frac{1}{2}\left(1-\boldsymbol{p}^{\prime, "}{ }^{\prime \prime} \boldsymbol{p}_{0}\right)_{\left|p_{0}\right|=1}==\frac{1+(\boldsymbol{e} \boldsymbol{m})^{2} \mp 2(\boldsymbol{e} \boldsymbol{m})\left(\boldsymbol{e} \boldsymbol{p}_{0}\right)-\left[p_{0 x}-\boldsymbol{e}_{x}\left(\boldsymbol{e} \boldsymbol{p}_{0}\right)\right]^{2}-\left[p_{0 y}-\boldsymbol{e}_{y}\left(\boldsymbol{e} \boldsymbol{p}_{0}\right)\right]^{2}}{1+(\boldsymbol{e} \boldsymbol{m})^{2} \mp 2(\boldsymbol{e} \boldsymbol{m})\left(\boldsymbol{e} \boldsymbol{p}_{0}\right)} .
$$


If we multiply this expression by $\sigma^{\prime}$," from Eq. (89), we obtain the spin-flip cross sections for inelastic coherent one-magnon scattering $\left(\left|\boldsymbol{p}_{0}\right|=1\right)$

$$
\begin{array}{r}
\sigma_{\mathrm{f}}^{\prime{ }^{\prime \prime}}=\left[1+(\boldsymbol{e} \boldsymbol{m})^{2} \mp 2(\boldsymbol{e} \boldsymbol{m})\left(\boldsymbol{e} \boldsymbol{p}_{0}\right)-\left(\boldsymbol{X} \boldsymbol{p}_{0}\right)^{2}-\left(\boldsymbol{Y} \boldsymbol{p}_{0}\right)^{2}\right]\left(r_{0} \gamma_{N}\right)^{2} F^{2}(\boldsymbol{q}) \frac{1}{2} S \exp \left\{-2 W_{\boldsymbol{q}}\right\} \\
\cdot \sum_{\tau} \int \mathrm{d}^{3} k\left(p^{\prime} / p\right)\left[n(\boldsymbol{k})+\frac{1}{2} \pm \frac{1}{2}\right] \delta(\boldsymbol{q} \mp \boldsymbol{k}-\boldsymbol{\tau}) \delta\left(E_{p^{\prime}}-E_{p} \pm \varepsilon_{\boldsymbol{k}}\right) .
\end{array}
$$

Multiplying Eq. (89) by $1-Q^{\prime}$," yields the non-flip cross sections for inelastic scattering $\left(\left|\boldsymbol{p}_{0}\right|=1\right)$

$$
\begin{aligned}
& \sigma_{\mathrm{f}}^{\prime \prime \prime}=\left[\left(\boldsymbol{X} \boldsymbol{p}_{0}\right)^{2}+\left(\boldsymbol{Y} \boldsymbol{p}_{0}\right)^{2}\right]\left(r_{0} \gamma_{\mathrm{N}}\right)^{2} F^{2}(\boldsymbol{q}) \frac{1}{2} S \exp \left\{-2 W_{\boldsymbol{q}}\right\} \\
& \cdot \sum_{\tau} \int \mathrm{d}^{3} k\left(p^{\prime} / p\right)\left[n(\boldsymbol{k})+\frac{1}{2} \pm \frac{1}{2}\right] \delta(\boldsymbol{q} \mp \boldsymbol{k}-\boldsymbol{\tau}) \delta\left(E_{p^{\prime}}-E_{p} \pm \varepsilon_{\boldsymbol{k}}\right) .
\end{aligned}
$$

As before, the primed quantities refer to scattering with emission of a magnon (in which case the upper signs are applicable).

The angular dependence of $\sigma_{\mathrm{f}}^{\prime \prime \prime}$ and $\sigma_{\text {nff }}^{\prime \prime \prime}$ is the same as for the corresponding nuclear magnetic quantities in Eqs. (24) - (29) if we put $\boldsymbol{p}_{0}= \pm \boldsymbol{m}$. Indeed, for $\boldsymbol{p}_{0}=\boldsymbol{m}$ we obtain from Eqs. (92) and (93)

$$
\sigma_{\mathrm{f}}^{\prime \prime \prime} \sim\left(1 \mp \cos ^{2} \theta\right)^{2} ; \sigma_{\mathrm{nf}}^{\prime \prime \prime} \sim \sin ^{2} 2 \theta
$$

where $\theta$ is the angle of the scattering vector $\boldsymbol{q}$ with the $z$-axis.

The total spin-flip probability $Q$, which corresponds to the total (elastic and inelastic) scattering, can be calculated as follows

$$
\begin{aligned}
& Q=\sigma_{\mathrm{f}} / \sigma, \sigma=\sigma_{\mathrm{nf}}+\sigma_{\mathrm{f}}, \\
& \sigma_{\mathrm{nf}}=\sigma_{\mathrm{nf}}^{0}+\sigma_{\mathrm{nf}}^{\prime(\mathrm{m})}+\sigma_{\mathrm{nf}}^{\prime \prime(\mathrm{m})}+\sigma_{\mathrm{fn}}^{\prime(\mathrm{n})}+\sigma_{\mathrm{nf}}^{\prime \prime(\mathrm{n})} ; \\
& \sigma_{\mathrm{f}}=\sigma_{\mathrm{f}}^{0(\mathrm{~m})}+\sigma_{\mathrm{f}}^{(\prime \mathrm{m})}+\sigma_{\mathrm{f}}^{\prime \prime(\mathrm{m})}+\sigma_{\mathrm{f}}^{\prime(\mathrm{n})}+\sigma_{\mathrm{f}}^{\prime \prime(\mathrm{n})} .
\end{aligned}
$$

The elastic cross sections $\sigma_{\mathrm{f}}^{0}$ and $\sigma_{\mathrm{nf}}^{0}$ are given by Eqs. (86) and (87). They include all types of scattering: nuclear, magnetic and nuclear magnetic, as well as the inteference terms. The magnetic cross sections $\sigma_{\mathrm{f}, \mathrm{nf}}^{\prime(\mathrm{m})}$ and $\sigma_{\mathrm{f}, \mathrm{nf}}^{\prime \prime(m)}$ are given by Eqs. (92) and (93). The coresponding nuclear and nuclear magnetic cross sections $\sigma_{\mathrm{f}, \mathrm{nf}}^{\prime(\mathrm{n})}$ and $\sigma_{\mathrm{f}, \mathrm{nf}}^{\prime \prime}(\mathrm{n})$ are easily extracted from Eqs. (33) and (35).

\subsection{The Temperature-Dependence of Neutron Scattering without Spin-Recoil}

The zero-magnon probability $\varkappa$ which was defined in Sect. 2.3 is proportional to $S^{2}(T)$. It thus has the same temperature-dependence as the square of the magnetization in the ferro-magnetic scatterer ${ }^{9}$. From Eqs. (71), (72) and (75) we get

$$
\begin{aligned}
S^{2}(T) \equiv S^{2}-(2 S / N) & \sum_{\boldsymbol{k}} n(\boldsymbol{k}) \\
= & S^{2}-2 S \frac{V_{0}}{(2 \pi)^{3}} \int \frac{\mathrm{d}^{3} k}{\exp \left\{\beta D k^{2}\right\}-1}
\end{aligned}
$$

where we have put $2 S J_{0} a^{2}=D$ in Eq. (45) and $\beta=1 / k_{\mathrm{B}} T$ as in Eq. (48). At low temperatures $\left(\beta D k_{\max }^{2} \gg 1\right)$ the upper limit of the integral in Eq. (96) can be extended to infinity

$$
\begin{array}{r}
S^{2}(T)=S^{2}-2 S \frac{V_{0}}{(2 \pi)^{3}}\left(\frac{1}{\beta D}\right)^{3 / 2} \int \frac{x^{1 / 2} \mathrm{~d} x}{e^{x}-1} \\
=S^{2}-0,117 S\left(k_{\mathrm{B}} T / 2 J_{0} S\right)^{3 / 2} .
\end{array}
$$

Here we have inserted the numerical value of the integral in terms of the $\Gamma$-function and of the Riemann $\zeta$-function, $\Gamma\left(\frac{3}{2}\right) \zeta\left(\frac{3}{2}, 1\right)$, supposing $V_{0}=a^{3}$. Finally we obtain the zero-magnon probability

$$
\begin{aligned}
\varkappa & =\left[S^{2}(T)\right] /[S(S+1)] \\
& =\left[S-0,117\left(k_{\mathrm{B}} T / 2 J_{0} S\right)^{3 / 2}\right] /[S+1] \\
& =1-\left[1+0,117\left(k_{\mathrm{B}} T / 2 J_{0} S\right)^{3 / 2}\right] /[S+1] .
\end{aligned}
$$

The second term in the last expression is the probability for excitation of a spin-wave by the scattering neutron. Its dependence on temperature is the same as that of the magnetization in the ferro-magnetic scatterer.

The zero-magnon probability for spin-flip scattering is given by Eq. (77) with Eqs. (85) and (95). The factor $Q^{0} / Q$ does not change the temperaturedependence significantly.

\section{Recoilless Spin-Flip in Antiferromagnets and Ferromagnetic Spirals}

Let us consider a simple antiferromagnet consisting of two sublattices. Equation (56) is applicable for the summation in each sublattice. If both sublattices consist of the same type of atoms, the magnetic unit cell will be two times greater than the chemical cell. Denoting by $V_{0 \mathrm{~m}}$ the volume of the magnetical unit cell, by $\tau_{\mathrm{m}} / 2 \pi$ a vector of the corre-

9 C. KitTel, Quantum Theory of Solids, Wiley \& Sons, New York 1963. 
sponding reciprocal lattice and by $\boldsymbol{r}_{12}$ the vector linking one atom to a neighboring atom of the second sublattice, we obtain, using the notation of Sect. 2.5,

$$
\begin{gathered}
\sigma_{\mathrm{f}}^{0}=2 B^{2}\left[M^{2}-\left(\boldsymbol{M} \boldsymbol{p}_{0}\right)\right]\left(1-\cos \boldsymbol{q} \boldsymbol{r}_{12}\right) \\
\cdot\left[(2 \pi)^{3} / V_{0 \mathrm{~m}}\right] \cdot \exp \left\{-2 W_{\boldsymbol{q}}\right\} \cdot \sum_{\tau_{\mathrm{m}}} \delta\left(\boldsymbol{q}-\boldsymbol{\tau}_{\mathrm{m}}\right), \\
\sigma_{\mathrm{nf}}^{0}=2\left[A^{2}\left(1+\cos \boldsymbol{q} \boldsymbol{r}_{12}\right)+B^{2}\left(1-\cos \boldsymbol{q} \boldsymbol{r}_{12}\right) \boldsymbol{M} \boldsymbol{p}_{0}\right] \\
\cdot\left[(2 \pi)^{3} / V_{0 \mathrm{~m}}\right] \cdot \exp \left\{-2 W_{\boldsymbol{q}}\right\} \cdot \sum_{\tau_{\mathrm{m}}} \delta\left(\boldsymbol{q}-\boldsymbol{\tau}_{\mathrm{m}}\right) .
\end{gathered}
$$

These cross sections can be compared to the corresponding results in ferromagnets, Eqs. (86) and (87), and also refer to one atom. The spin-flip probability for elastic scattering will be

$$
\begin{aligned}
Q_{0} & =\sigma_{\mathrm{f}}^{0} /\left(\sigma_{\mathrm{f}}^{0}+\sigma_{\mathrm{nf}}^{0}\right) \\
& =\left[M^{2}-\left(\boldsymbol{M} \boldsymbol{p}_{0}\right)^{2}\right]\left[\frac{A^{2}}{B^{2}} \frac{1+\cos \boldsymbol{q} \boldsymbol{r}_{12}}{1-\cos \boldsymbol{q} \boldsymbol{r}_{12}}+M^{2}\right]^{-1}
\end{aligned}
$$

At low temperatures inelastic scattering can be neglected and thus $Q_{0}$ can be identified with the r.s.f. scattering probability $Q$.

In a ferromagnetic spiral the orientations of the spins as one goes from an atom to the next change periodically and the period of this variation does not coincide with the period of the crystal lattice. This results in a helical structure

$$
\begin{array}{r}
\boldsymbol{S}_{j}=S_{0}{ }^{z} \boldsymbol{m}+\frac{1}{2} S_{0}{ }^{-} \exp \left\{-i \boldsymbol{k}_{0} \boldsymbol{R}_{j}\right\} \cdot \boldsymbol{m}^{+} \\
+\frac{1}{2} S_{0}{ }^{+} \exp \left\{i \boldsymbol{k}_{0} \boldsymbol{R}_{j}\right\} \cdot \boldsymbol{m}^{-},
\end{array}
$$

where $S_{0}{ }^{z}$ is the ferromagnetic component of the spiral, $S_{0}{ }^{+}$and $S_{0}^{-}$are also constants, $\boldsymbol{m}^{+}=\boldsymbol{u}_{x}+i \boldsymbol{u}_{y}$, $\boldsymbol{m}^{-}=\boldsymbol{u}_{x}-i \boldsymbol{u}_{y}$ and $\boldsymbol{u}_{x}, \boldsymbol{u}_{y}$ are unit vectors in the base plane. $\boldsymbol{k}_{0}$ and the unit vector $\boldsymbol{m}$ are directed along the axis of the helix, which is the $z$-axis. If we repeat the calculations of Sect. 2.5 , we obtain the elastic cross sections

$$
\sigma_{\mathrm{f}, \mathrm{nf}}^{0}=\sigma_{\mathrm{f}, \mathrm{nf}}^{01}+\sigma_{\mathrm{f}, \mathrm{nf}}^{02}+\sigma_{\mathrm{f}, \mathrm{nf}}^{03}
$$

where $\sigma_{\mathrm{f}}^{01}$ and $\sigma_{\mathrm{fn}}^{01}$ are again given by Eqs. (86) and (87) with $S^{2}(T)$ replaced by $\left(S_{0}{ }^{z}\right)^{2}$ in Eq. (82). The additional terms $\sigma_{\mathrm{f}}^{02} \mathrm{nf}$ and $\sigma_{\mathrm{f}, \mathrm{nf}}^{03}$ are characteristic of scattering in ferromagnetic spirals yielding a satellite maximum on each side of the usual mag-

10 C. Kittel, Rev. Mod. Phys. 21, 541 [1949]; Landolt BöRNSTEIN, „Zahlenwerte und Funktionen“, Vol. 2, part 9, Springer, Berlin 1962. netic maxima

$$
\begin{aligned}
\sigma_{\mathrm{f}}^{02,03}= & {\left[1+(\boldsymbol{e} \boldsymbol{m})^{2} \mp 2(\boldsymbol{e} \boldsymbol{m})\left(\boldsymbol{e} \boldsymbol{p}_{0}\right)\right.} \\
- & \left.\left(\boldsymbol{X} \boldsymbol{p}_{0}\right)^{2}-\left(\boldsymbol{Y} \boldsymbol{p}_{0}\right)^{2}\right] \cdot\left(r_{0} \gamma_{\mathrm{N}}\right)^{2} F^{2}(\boldsymbol{q})\left(S_{0}^{ \pm}\right)^{2} \\
& \cdot \frac{(2 \pi)^{3}}{V_{0}} \exp \{-2 W \boldsymbol{q}\} \cdot \sum_{\boldsymbol{\tau}} \delta(\boldsymbol{q} \mp \boldsymbol{k}-\boldsymbol{\tau}) . \\
\sigma_{\mathrm{nf}}^{02,03}= & {\left[\left(\boldsymbol{X} \boldsymbol{p}_{0}\right)^{2}+\left(\boldsymbol{Y} \boldsymbol{p}_{0}\right)^{2}\right] \cdot\left(r_{0} \gamma_{\mathrm{N}}\right)^{2} F^{2}(\boldsymbol{q})\left(S_{0}^{ \pm}\right)^{2} } \\
\cdot & \frac{(2 \pi)^{3}}{V_{0}} \exp \left\{-2 W_{\boldsymbol{q}}\right\} \sum_{\boldsymbol{\tau}} \delta(\boldsymbol{q} \mp \boldsymbol{k}-\boldsymbol{\tau}) \cdot
\end{aligned}
$$

Here we used the notations of Eqs. (90) - (93). The angular dependence is the same as for scattering with emission or absorption of a magnon with $\boldsymbol{k}=\boldsymbol{k}_{\mathbf{0}}$. From Eqs. (103) - (105) we obtain for low temperature the r.s.f. scattering probability

$$
Q=\left(\sigma_{\mathrm{f}}^{01}+\sigma_{\mathrm{f}}^{02}+\sigma_{\mathrm{f}}^{03}\right) /\left(\sigma_{\mathrm{f}}^{0}+\sigma_{\mathrm{nf}}^{0}\right) .
$$

\section{Conclusion}

We have seen that generally magnetic and nuclear magnetic recoilless spin-flip scattering is possible. R.s.f. scattering is an elastic process which occurs when the angular momentum involved in the spinflip is absorbed by the crystal as a whole. The corresponding spin-flip and zero-magnon probabilities $Q^{0}$ and $\varkappa$ are functions of temperature.

As an example we shall calculate them for scattering in iron. There will be no nuclear magnetic scattering in this case, as the nuclear spin is zero. The total electronic spin is 1 for iron and the interaction integral ${ }^{10} J_{0} \approx 160 k_{\mathrm{B}}$ in Eq. (65), where $k_{\mathrm{B}}$ is the Boltzmann constant. Using Eqs. (82), (85) and (98) we obtain the following values for $\boldsymbol{p}_{0}=\boldsymbol{m}$ and $\theta=\pi / 4$

\begin{tabular}{lcccc}
\hline$T{ }^{\circ} \mathrm{K}$ & 0 & 80 & 293,2 & 640 \\
\hline$Q \circ \%$ & 4,64 & 4,61 & 4,29 & 3,51 \\
$\varkappa \%$ & 50,0 & 49,3 & 44,8 & 34,7 \\
$\varkappa Q \circ \%$ & 2,32 & 2,27 & 1,92 & 1,18 \\
\hline
\end{tabular}

The product $\varkappa Q^{0}$ is the fraction of r.s.f. scattering in the total scattering. For example at $0{ }^{\circ} \mathrm{K}$, only $2.32 \%$ of the total scattering or $4.64 \%$ of the elastic scattering is r.s.f. scattering, although $50 \%$ of the total scattering does not involve emission or absorption of magnons.

I am grateful to Professor H. Maier-Leibnitz who suggested and encouraged this work. I thank Dr. H. SCHMIDT for many fruitful discussions and valuable advices, and Dr. J. KANE for a critical reading of the manuscript. 